\title{
The conformal measures of a normal subgroup of a cocompact Fuchsian group
}

\author{
OFER SHWARTZ \\ Technion - Israel Institute of Technology, Haifa, Israel \\ (e-mail:ofer12@yahoo.com)
}

(Received 2 December 2019 and accepted in revised form 22 June 2020)

Abstract. In this paper we study the conformal measures of a normal subgroup of a cocompact Fuchsian group. In particular, we relate the extremal conformal measures to the eigenmeasures of a suitable Ruelle operator. Using Ancona's theorem, adapted to the Ruelle operator setting, we show that if the group of deck transformations $G$ is hyperbolic then the extremal conformal measures and the hyperbolic boundary of $G$ coincide. We then interpret these results in terms of the asymptotic behavior of cutting sequences of geodesics on a regular cover of a compact hyperbolic surface.

Key words: conformal measures, regular covers, Fuchsian groups, Ruelle operator, Martin boundary.

2020 Mathematics Subject Classification: 37D40 (Primary); 37F32 (Secondary)

\section{Introduction}

Let $\mathbb{D}=\{z \in \mathbb{C}:|z|<1\}$ be the open hyperbolic unit disc and let $\partial \mathbb{D}=\{z \in \mathbb{C}:|z|=$ $1\}$. Let $\Gamma$ be a Fuchsian group (a discrete subgroup of Möbius transformations) which preserves $\mathbb{D}$. We denote by $\delta_{\Gamma}$ the critical exponent of $\Gamma$ (see the definition in $\S 2.5$ ). Given $\delta>0$, a finite measure $\mu$ on $\partial \mathbb{D}$ is said to be $(\Gamma, \delta)$-conformal if for every $\gamma \in \Gamma$,

$$
\frac{d(\mu \circ \gamma)}{d \mu}=\left|\gamma^{\prime}\right|^{\delta}
$$

We denote by $\operatorname{Conf}(\Gamma, \delta)$ the collection of $(\Gamma, \delta)$-conformal measures and by $\operatorname{ext}(\operatorname{Conf}(\Gamma, \delta))$ the extremal points of $\operatorname{Conf}(\Gamma, \delta)$.

Conformal measures have many applications in hyperbolic geometry. Let $\mu$ and $\mu^{\prime}$ be two $(\Gamma, \delta)$-conformal measures. Then the measure

$$
\frac{d \mu\left(\xi^{-}\right) d \mu^{\prime}\left(\xi^{+}\right) d t}{\left\|\xi^{-}-\xi^{+}\right\|^{2 \delta}}
$$


on $\partial \mathbb{D}^{2} \times \mathbb{R}$ projects to a geodesic-flow-invariant measure on $T^{1}(\mathbb{D} / \Gamma$ ) (the unit tangent bundle of $\mathbb{D} / \Gamma$ ), see [6]; the measure

$$
e^{\delta s} d \mu(\xi) d s d t
$$

on $\partial \mathbb{D} \times \mathbb{R}^{2}$ projects to a horocycle-flow-invariant measure on $T^{1}(\mathbb{D} / \Gamma)$ and the function

$$
h(z)=\int_{\partial \mathbb{D}}\left(\frac{1-|z|^{2}}{|\xi-z|^{2}}\right)^{\delta} d \mu(\xi), \quad z \in \mathbb{D},
$$

is a positive $\Gamma$-invariant $\delta(\delta-1)$-eigenfunction of the Laplacian Beltrami operator. Moreover, every such eigenfunction arises in that way; see [5, 31].

If the underlying surface $\mathbb{D} / \Gamma$ is a tame surface then every ergodic horocycle-flowinvariant Radon measure which is not supported on a single horocycle is of the form of equation (1); see [43]. See also [32] for a similar decomposition in higher dimensions.

The existence of a $(\Gamma, \delta)$-conformal measure was first proven by Patterson [37] for the critical value $\delta=\delta_{\Gamma}$ and by Sullivan in higher dimensions [52]. Later on, in [53] Sullivan showed that for non-cocompact groups with no parabolic elements, a $(\Gamma, \delta)$-conformal measure exists if and only if $\delta \geq \delta_{\Gamma}$. In [40], Roblin studied the conformal measures in more general settings via a Martin boundary approach. A more general class of measures, quasiconformal measures, has been considered as well; see [9, 14].

Furstenberg [22] showed that if $\Gamma$ is cocompact (i.e. $\mathbb{D} / \Gamma$ is compact) then the Lebesgue measure is the unique $\left(\Gamma, \delta_{\Gamma}\right)$-conformal measure and there are no other $(\Gamma, \delta)$-conformal measures for all $\delta>\delta_{\Gamma}$. Variants of this result were proven by Dani [16] for cofinite groups and by Burger [12] for geometrically finite groups. Their original motivation was the classification of the horocycle-flow-invariant measures. See also [28] for a study of the ergodicity of the horocycle flow with respect to the Liouville measure and [39] for an extensive study of the conformal measures in negatively curved geometrically finite manifolds.

In this work we study the conformal measures of a normal subgroup of a cocompact Fuchsian group, namely under the assumption that there exists a cocompact Fuchsian group $\Gamma_{0}$ with $\Gamma \triangleleft \Gamma_{0}$. In particular, we focus on the classification of the conformal measures and their identification with some structure of the group of deck transformations $G \cong \Gamma_{0} / \Gamma$. To the best of our knowledge, such an identification is known only when the group $G$ is a nilpotent group. Then the extremal conformal measures are identified with homomorphisms of the form $\varphi: G \rightarrow \mathbb{R}$; see [33, 34]. Another related work is [45], where Schapira and Sarig studied the generic points of the horocycle-flow-invariant measures on $\mathbb{Z}^{d}$-covers (namely $G \cong \mathbb{Z}^{d}$ ) in terms of the almost sure asymptotic velocity of geodesics.

1.1. Conformal measures and eigenmeasures of the Ruelle operator. In $\S 3$ we show that for every $\delta \geq \delta_{\Gamma}$ there is a linear one-to-one correspondence between the extremal $(\Gamma, \delta)$-conformal measures and eigenmeasures of a suitable Ruelle operator. As the theory of the eigenmeasures of the Ruelle operator is well developed (see $[10,18,35,41,42$, 49, 51]), this correspondence can be a step towards a classification of the conformal measures. In particular, in [49] the author presented the eigenmeasures of a transient Ruelle operator (see the definitions in §2.2) in terms of points on a Martin boundary. Thus, 
the classification of the conformal measures directly translates to the identification of the Martin boundary.

The correspondence is stated using the Bowen-Series coding. In more detail, for a cocompact Fuchsian group $\Gamma_{0}$ let $F_{0} \subseteq \mathbb{D}$ be a fundamental domain for $\mathbb{D} / \Gamma_{0}$ with even corners, that is, $F_{0}$ is a union of complete geodesics in $\mathbb{D}$. Every compact surface $\mathbb{D} / \Gamma_{0}$ has such fundamental domains; see, for example, [7]. For a set $A$, we write $\operatorname{int}(A)=$ $A \backslash \partial A$. In [11], Bowen and Series constructed (with respect to $F_{0}$ ) a partition $\left\{I_{a}\right\}_{a \in S_{0}}$ of $\partial \mathbb{D}$ into closed arcs with disjoint interiors and a set $\left\{e_{a}\right\}_{a \in S_{0}} \subseteq \Gamma_{0}$ such that the set $\left\{e_{a}\right\}_{a \in S_{0}}$ generates $\Gamma_{0}$ and the Bowen-Series map $f_{\Gamma_{0}}: \partial \mathbb{D} \rightarrow \partial \mathbb{D}$,

$$
f_{\Gamma_{0}}(\xi)=e_{a}^{-1} \xi, \quad \xi \in \operatorname{int}\left(I_{a}\right),
$$

induces a Markov partition of $\partial \mathbb{D}$, namely the space

$$
\Sigma:=\left\{\left(\sigma_{i}\right): \forall i \geq 0, \sigma_{i} \in S_{0} \text { and } \operatorname{int}\left(f_{\Gamma_{0}}\left(I_{\sigma_{i}}\right)\right) \cap \operatorname{int}\left(I_{\sigma_{i+1}}\right) \neq \varnothing\right\}
$$

along with the left-shift transformation is a topological Markov shift (see §2.1). Since the group $\Gamma_{0}$ has no parabolic elements the set $S_{0}$ is finite and $(\Sigma, T)$ is actually a subshift of finite type. Let $\pi_{\Sigma}: \Sigma \rightarrow \partial \mathbb{D}$ be the canonical projection, $\pi_{\Sigma}(\sigma) \in \bigcap_{n \geq 0} f_{\Gamma_{0}}^{-n} I_{\sigma_{n}}$ (the intersection is a singleton; see [6]). For several other important properties of the Bowen-Series coding, see $\$ 2.6$.

Let $(X, T)$ be the group extensionof $\Sigma$ with $G=\Gamma_{0} / \Gamma$ and let $T: X \rightarrow X$ be the left-shift transformation; see $\S 2.6$. We sometime use the canonical correspondence and identify $X$ with $\Sigma \times G$. Given $\delta>0$, let $\phi^{X, \delta}: X \rightarrow \mathbb{R}$,

$$
\phi^{X, \delta}(\sigma, \gamma \Gamma):=-\delta \log \left|\left(e_{\sigma_{0}}^{-1}\right)^{\prime}\left(\pi_{\Sigma} \circ \pi_{X}(x)\right)\right|
$$

where $\pi_{X}: X \rightarrow \Sigma$ is the natural projection from $X$ to $\Sigma$. The Ruelle operator associated to $\phi^{X, \delta}$, evaluated on a function $f: X \rightarrow \mathbb{R}$ and a point $x \in X$, is

$$
\left(L_{\phi^{X, \delta}} f\right)(x)=\sum_{y: T y=x} e^{\phi^{X, \delta}(y)} f(y) ;
$$

see Definition 2.1. In $\S 3$ we prove the following theorem which connects between the conformal measures and the eigenmeasures of $L_{\phi^{X, \delta}}$.

THEOREM 1.1. Let $\Gamma_{0}$ be a cocompact Fuchsian group, let $\Gamma \triangleleft \Gamma_{0}$ and let $\delta \geq \delta_{\Gamma}$. Then the following mapping $\psi$ is an affine bijection between the Radon eigenmeasures of $L_{\phi^{X, \delta}}$ for the eigenvalue 1 and the $(\Gamma, \delta)$-conformal measures: for a Radon eigenmeasure $\mu_{X}$ and a Borel set $E \subseteq \partial \mathbb{D}$,

$$
\psi\left(\mu_{X}\right)(E)=\mu_{X}\left(\pi_{\Sigma}^{-1}(E) \times\{\Gamma\}\right) .
$$

1.2. Application to hyperbolic covers. In $\S \S 4$ and 5 we apply the principle described above to the case where the group of deck transformations $G=\Gamma_{0} / \Gamma$ is hyperbolic. In the canonical probabilistic setting, Ancona's well-known theorem [3, 4] relates the Martin boundary of a finite-range random walk on a hyperbolic graph to the hyperbolic boundary of the graph. In his original work, Ancona actually studied elliptic operators; see [54] 
for the probabilistic interpretation. Using an extended version of Ancona's theorem to the Ruelle operator setting (see $\$ 2.4$ ), for every $\delta>\delta_{\Gamma}$ we relate the $(\Gamma, \delta)$-conformal measures, via a suitable Martin boundary, to the hyperbolic boundary of $G$, denoted by $\partial G$.

In what follows, a sequence $\left(a_{i}\right)$ with $a_{i} \in S_{0}$ is called a boundary expansion of a point $\xi \in \partial \mathbb{D}$ if for every $n \geq 0, f_{\Gamma_{0}}^{n}(\xi) \in I_{a_{n}}$. Observe that $\left(a_{i}\right)$ is a boundary expansion of a point $\xi \in \partial \mathbb{D}$ if and only if $\pi_{\Sigma}\left(a_{0}, a_{1}, \ldots\right)=\xi$.

THEOREM 1.2. Let $\Gamma_{0}$ be a cocompact Fuchsian group, let $\Gamma \triangleleft \Gamma_{0}$ and let $\delta>\delta_{\Gamma}$. Assume that $G=\Gamma_{0} / \Gamma$ is a hyperbolic group. Then, for every $\mu \in \operatorname{Conf}(\Gamma, \delta)$, for $\mu$-almost every (a.e.) $\xi \in \partial \mathbb{D}$ with Bowen-Series coding $\left(a_{n}\right)$, the sequence

$$
e_{a_{n}}^{-1} \ldots e_{a_{0}}^{-1} \Gamma
$$

converges to a point in $\partial G$. If $\mu \in \operatorname{ext}(\operatorname{Conf}(\Gamma, \delta))$, then there exists $\eta \in \partial G$ such that the sequence almost surely converges to $\eta$. Conversely, for every $\eta \in \partial G$, there exists a unique $\mu \in \operatorname{ext}(\operatorname{Conf}(\Gamma, \delta))$ with $\eta$ its almost surely limiting point of the sequence.

We interpret the result of Theorem 1.2 in terms of cutting sequences of geodesics as well. In more detail, let

$$
\mathcal{R}=\left\{\left(\xi^{-}, \xi^{+}\right) \in(\partial \mathbb{D})^{2}: \text { the geodesic curve between } \xi^{-} \text {and } \xi^{+} \text {intersects int } F_{0}\right\}
$$

Recall that for every $\gamma_{1}, \gamma_{2} \in \Gamma_{0}, \operatorname{int}\left(\gamma_{1} F_{0}\right) \cap \operatorname{int}\left(\gamma_{2} F_{0}\right) \neq \varnothing$ if and only if $\gamma_{1}=\gamma_{2}$ and that the copies $\gamma_{1} F_{0}, \gamma_{2} F_{0}$ share a common edge if and only if $\gamma_{1} \gamma_{2}^{-1} \in\left\{e_{a}\right\}_{a \in S_{0}}$. Given $\left(\xi^{-}, \xi^{+}\right) \in \mathcal{R}$, let $\left(F_{i}\right)_{i \in \mathbb{Z}}$ be the sequence of copies of $F_{0}$ that the geodesic curve between $\xi^{-}$and $\xi^{+}$intersects. If the curve passes through a vertex of some $F_{i}$, we perturb the curve around it; see [48, Figure 5]. Then, for all $i$, there exists a unique $e_{i} \in\left\{e_{a}\right\}_{a \in S_{0}}$ such that $F_{i}=e_{i}^{-1} F_{i+1}$. The sequence $\left(e_{i}\right)$ is called the cutting sequence of $\left(\xi^{-}, \xi^{+}\right)$. For $\left(\xi^{-}, \xi^{+}\right) \in \mathcal{R}$ with a cutting sequence $\left(e_{i}\right)$, we write

$$
\eta_{n}^{+}\left(\xi^{-}, \xi^{+}\right):=e_{n}^{-1} \cdots e_{0}^{-1} \Gamma
$$

and

$$
\eta_{n}^{-}\left(\xi^{-}, \xi^{+}\right):=\left(e_{-n-1}\right)^{-1} \cdots\left(e_{-1}\right)^{-1} \Gamma .
$$

In $\S 5$ we prove the following theorem which describes the almost surely limiting behavior of $\eta_{n}^{+}$and $\eta_{n}^{-}$.

THEOREM 1.3. Let $\Gamma_{0}$ be a cocompact Fuchsian group, let $\Gamma \triangleleft \Gamma_{0}$, let $\delta>\delta_{\Gamma}$ and let $\mu \in$ $\operatorname{Conf}(\Gamma, \delta)$. Assume that $G=\Gamma / \Gamma_{0}$ is a hyperbolic group. Then the following statements hold.

(1) For $\mu$-a.e. $\xi^{+} \in \partial \mathbb{D}$, for all but countably many $\xi^{-} \in \partial \mathbb{D}$ such that $\left(\xi^{-}, \xi^{+}\right) \in \mathcal{R}$, the sequence $\eta_{n}^{+}\left(\xi^{-}, \xi^{+}\right)$converges to a point in $\partial G$.

(2) For $\mu$-a.e. $\xi^{-} \in \partial \mathbb{D}$, for all but countably many $\xi^{+} \in \partial \mathbb{D}$ such that $\left(\xi^{-}, \xi^{+}\right) \in \mathcal{R}$, the sequence $\eta_{n}^{-}\left(\xi^{-}, \xi^{+}\right)$converges to a point in $\partial G$. 
If $\mu \in \operatorname{ext}(\operatorname{Conf}(\Gamma, \delta))$ then there exists a point $\eta \in \partial G$ such that the sequences almost surely converge to $\eta$. Conversely, for every $\eta \in \partial G$, there exists a unique $\mu \in \operatorname{ext}(\operatorname{Conf}(\Gamma, \delta))$ with $\eta$ its almost surely limiting point of the sequences.

The limiting point $\eta$ from Theorem 1.3 is the same limiting point from Theorem 1.2. We emphasize that Theorem 1.3 does not follow directly from Theorem 1.2 because when $\Gamma_{0}$ is cocompact the set of cutting sequences is not a Markov shift; see [48]. However, as was shown by Series in [48] and also by Adler and Flatto in [2], the cutting sequences and boundary expansions (namely the two-sided Bowen-Series coding) are conjugate; see $\S 5$. Although Theorems 1.2 and 1.3 are equivalent in some sense, for completeness of this paper, a rigorous proof for the latter theorem is included in $§ 5$.

The extended Ancona's theorem (see Theorem 2.9) holds only for supercritical values, which translates in this setting to $\delta>\delta_{\Gamma}$. Recently and independently, Bispo and Stadlbauer [8] showed that for a potential function with a quasi-symmetric Green's function on a group extension of a hyperbolic group, the results of the extended Ancona's theorem also hold at the critical value. In particular, they showed that the results of Theorems 1.2 and 1.3 hold at the critical value $\delta=\delta_{\Gamma}$ as well. Moreover, using the symbolic coding presented recently in [13], they derived a similar correspondence between the conformal measures and the hyperbolic boundary which holds at critical value $\delta=\delta_{\Gamma}$ for regular covers of $C A T(-1)$-spaces with compact convex core and hyperbolic group of deck transformations.

For similar results on dependent random walks involving invariant measures (rather than conformal measures), see $[29,30]$.

\section{Preliminaries}

2.1. Topological Markov shifts and the Ruelle operator. Let $S$ be an infinite countable set of states and let $\mathbb{A}=\left(\mathbb{A}_{a, b}\right)_{S \times S} \in\{0,1\}^{S \times S}$ be a transition matrix over $S$. For a subset $A \subseteq \mathbb{Z}$ and a vector $x \in S^{A}$, we denote by $x_{i}$ the $i$ th coordinate of $x$.

The (positive) one-sided topological Markov shift (TMS) is the space

$$
X=\left\{x \in S^{\mathbb{N} \cup\{0\}}: \mathbb{A}_{x_{i}, x_{i+1}}=1, \forall i \geq 0\right\}
$$

with the transformation $T: X \rightarrow X,(T x)_{i}=x_{i+1}$ and the metric

$$
d(x, y)=2^{-\inf \left\{i \geq 0: x_{i} \neq y_{i}\right\}} .
$$

If $\sum_{b} \mathbb{A}_{a, b}<\infty$ for every $a \in S$, then the space $(X, d)$ is locally compact and all cylinder sets

$$
\left[a_{0}, \ldots, a_{m}\right]:=\left\{x \in X: x_{i}=a_{i}, 0 \leq i \leq m\right\}
$$

are compact. A word $\left(a_{1}, \ldots, a_{n}\right) \in S^{n}$ is called admissible if $\left[a_{1}, \ldots, a_{n}\right] \neq \varnothing$. We denote by $\mathcal{W}^{n}$ the set of all admissible words of length $n$,

$$
\mathcal{W}^{n}=\left\{\left(a_{1}, \ldots, a_{n}\right) \in S^{n}:\left[a_{1}, \ldots, a_{n}\right] \neq \varnothing\right\} .
$$

We say that $X$ is topologically transitive, or simply transitive, if for every $a, b \in S$ there exists $n \geq 0$ such that $T^{-n}[a] \cap[b] \neq \varnothing$. 
We denote by $C_{c}(X)$ the space of all continuous functions from $X$ to $\mathbb{R}$ with compact support, by $C^{+}(X)$ the space of all non-negative continuous functions and by $C_{c}^{+}(X)=$ $C^{+}(X) \cap C_{c}(X)$ the space of all non-negative continuous functions with compact support.

The $m$-th variation of a function $\phi: X \rightarrow \mathbb{R}$ is

$$
\operatorname{Var}_{m}(\phi)=\sup \left\{|\phi(x)-\phi(y)|: x, y \in X, x_{i}=y_{i}, 0 \leq i<m-1\right\} .
$$

A function $\phi$ is said to have summable variations if $\sum_{m \geq 2} \operatorname{Var}_{m}(\phi)<\infty$. Notice that this condition is satisfied by all Hölder-continuous functions. We let $\phi_{n}=\sum_{i=0}^{n-1} \phi \circ T^{i}$ and $C_{\phi}=\sum_{m \geq 2} \operatorname{Var}_{m}(\phi)$.

Definition 2.1. The Ruelle operator $L_{\phi}$ evaluated on a function $f \in C(X)$ at a point $x \in X$ is

$$
\left(L_{\phi} f\right)(x)=\sum_{y: T y=x} e^{\phi(y)} f(y) .
$$

When $X$ is locally compact, the sum is finite for every $f \in C_{c}(X)$. Then, for every $n>0$,

$$
\left(L_{\phi}^{n} f\right)(x)=\sum_{y: T^{n} y=x} e^{\phi_{n}(y)} f(y) .
$$

Definition 2.2. The Gurevich pressure of $\phi$ is the limit

$$
P_{G}(\phi)=\limsup _{n \rightarrow \infty} \frac{1}{n} \log \sum_{T^{n} x=x} e^{\phi_{n}(x)} 1_{[a]}(x)
$$

for some $a \in S$ and $x \in X$.

If $(X, T)$ is topologically transitive and $\phi$ has summable variations, then $P_{G}(\phi)$ is independent of the choice of $a$; see [41]. When $P_{G}(\phi)<\infty$, we write $\rho(\phi)=\exp \left(P_{G}(\phi)\right)$.

2.2. The Martin boundary of a transient potential. Assume that $X$ is transitive and locally compact and that $\rho(\phi)<\infty$. Let $t \in[\rho(\phi), \infty)$. The $t$-Green function, evaluated at $f \in C_{c}(X)$ and $x \in X$, is

$$
G(f, x \mid t):=\sum_{n \geq 0} t^{-n}\left(L_{\phi}^{n} f\right)(x) .
$$

We say that $\phi$ is $t$-recurrent if $G(f, x \mid t)=\infty$ for some (or equivalently for every) $0 \not \equiv f \in$ $C_{c}^{+}(X)$ and $x \in X$. Otherwise, we say that $\phi$ is t-transient. If $\phi$ is 1 -transient, we simply say that it is transient. Then we write $G(f, x):=G(f, x \mid 1)$. Notice that the 'transience' in [42] means in our terminology $\rho(\phi)$-transience.

For a $t$-transient potential with summable variations, the author introduced in [49] a Martin boundary which represents all eigenmeasures (and analogously all eigenfunctions) of the Ruelle operator, for the eigenvalue $t$. We briefly describe the construction here. 
Fix $o \in S$ arbitrarily. When $\phi$ is $t$-transient, for every fixed $f \in C_{c}^{+}(X)$, the Martin kernel

$$
K(f, x \mid t):=\frac{G(f, x \mid t)}{G\left(1_{[o]}, x \mid t\right)}
$$

is continuous and bounded as a function of $x$. Let $\left\{w_{i}\right\}_{i \in \mathbb{N}}$ be an enumeration of $\bigcup_{i \geq 1} \mathcal{W}_{i}$. We define a new metric on $X$,

$$
\varrho(x, y \mid t)=\sum_{i=1}^{\infty} \frac{\left|K\left(1_{\left[w_{i}\right]}, x \mid t\right)-K\left(1_{\left[w_{i}\right]}, y \mid t\right)\right|+\left|1_{\left[w_{i}\right]}(x)-1_{\left[w_{i}\right]}(y)\right|}{\max _{z \in\left[w_{i}\right]}\left|K\left(1_{\left[w_{i}\right]}, z \mid t\right)\right|} .
$$

The $t$-Martin compactification, denoted by $\widehat{X}(t)$, is the completion of $X$ with respect to the metric $\varrho$. The $t$-Martin boundary, denoted by $\mathcal{M}(t)$, is the set of all new obtained points, $\mathcal{M}(t)=\widehat{X}(t) \backslash X$. For every fixed $f \in C_{c}(X)$, the Martin kernel $K(f, \cdot \mid t)$ is a $\varrho$-continuous function in $X$ and it can be uniquely extended to a $\varrho$-continuous function in $\hat{X}(t)$ via

$$
K(f, \omega \mid t)=\lim _{x \rightarrow \omega} K(f, x \mid t), \quad \omega \in \mathcal{M}(t) .
$$

Given $\omega \in \mathcal{M}(t)$ and $f \in C_{c}(X)$, we let $\mu_{\omega}(f):=K(f, \omega \mid t)$. Observe that for every $\omega \in$ $\mathcal{M}(t)$, the measure $\mu_{\omega}$ is a $t$-eigenmeasure of $L_{\phi}$.

The $t$-minimal boundary $\mathcal{M}_{m}(t)$ is the set of all points $\omega \in \mathcal{M}(t)$ such that the resulting measure $\mu_{\omega}$ is extremal in the cone of eigenmeasures for eigenvalue $t$. Then, for every positive Radon measure $\mu$ with $L_{\phi}^{*} \mu=t \mu$, there exists a unique finite measure $v$ on $\mathcal{M}_{m}(t)$ such that

$$
\mu(f)=\int_{\mathcal{M}_{m}(t)} \mu_{\omega}(f) d \nu(\omega) \text { for all } f \in C_{c}(X) .
$$

By definition, a sequence $x^{n} \in X$ converges to a point $\omega \in \mathcal{M}(t)$ in the topology of $\widehat{X}(t)$ if and only if $K\left(f, x^{n} \mid t\right) \underset{n \rightarrow \infty}{\longrightarrow} K(f, \omega \mid t)$ for all $f \in C_{c}(X)$. In particular, a point $\omega \in \mathcal{M}_{m}(t)$ is fully characterized by the following convergence property: for $\mu_{\omega}$-a.e. $x \in$ $X, T^{n} x \rightarrow \omega$ in $\hat{X}(t)$.

It is elementary to show that observing the first coordinate alone is sufficient to determine whether a sequence of internal points $x^{n} \in X$ converges to a boundary point $\omega \in \mathcal{M}_{m}(\lambda)$ : if $T^{n} x \rightarrow \omega$ and $x_{n}=y_{n}$ for all $n \geq 1$ then $T^{n} y \rightarrow \omega$ as well. In particular, we have the following proposition.

PROpOSITION 2.3. For every $\omega \in \mathcal{M}_{m}(\lambda)$ there is sequence $a_{n} \in[S]$ such that for every $x \in X$ with $x_{n} \in\left[a_{n}\right]$ we have that $T^{n} x \rightarrow \omega$.

In this paper we mainly assume that $P_{G}(\phi)<0$, which directly implies that $\phi$ is transient and the Martin boundary $\mathcal{M}:=\mathcal{M}(1)$ exists. We write $\mathcal{M}_{m}:=\mathcal{M}_{m}(1)$.

2.3. The hyperbolic boundary. We briefly recall the definitions of a hyperbolic graph and its boundary. For more detailed description, see [23] and also [54].

Let $E \subseteq S \times S$ be a set of edges over $S$. We say that $(S, E)$ is connected if for every $a, b \in S$, there exist $a_{1}, \ldots, a_{n} \in S$ such that $a_{1}=a, a_{n}=b$ and $\left(a_{i}, a_{i+1}\right) \in E$, 
$1 \leq i<n$. We say that $(S, E)$ is undirected if the set $E$ is symmetric. We say that $E$ is locally finite if for every $a \in S, \#\{b:(a, b) \in E\}<\infty$. We denote by $d_{E}(a, b)$ the length of a shortest path from $a$ to $b$ in $(S, E)$. When $(S, E)$ is undirected and connected, $d_{E}$ is a metric.

Definition 2.4. Let $(S, E)$ be a connected, undirected and locally finite graph. A geodesic triangle consists of three points $a, b, c \in S$ and three geodesic paths $\pi(a, b), \pi(b, c), \pi(c, a)$ from $a$ to $b, b$ to $c$, and $c$ to $a$, respectively. We say that the graph $(S, E)$ is $\delta$-hyperbolic if every geodesic triangle in the graph is $\delta$-thin, namely any point on one of its sides is at distance at most $\delta$ from the other two sides.

Let $(S, E)$ be a $\delta$-hyperbolic graph. For $a, b, o \in S$, let

$$
|a \wedge b|_{o}:=\frac{1}{2}\left(d_{E}(o, a)+d_{E}(o, b)-d_{E}(a, b)\right) .
$$

Fix an origin point $o \in S$.

Definition 2.5. We say that a sequence $a_{n} \in S$ converges to the hyperbolic boundary in $(S, E)$ if $\lim _{m, n \rightarrow \infty}\left|a_{n} \wedge a_{m}\right|_{o}=\infty$. Two sequences converging to the hyperbolic boundary $a_{n}, b_{n} \in S$ are said to be equivalent if

$$
\lim _{n \rightarrow \infty}\left|a_{n} \wedge b_{n}\right|_{o} \rightarrow \infty
$$

It is easy to verify that these definitions do not depend on $o \in S$.

Definition 2.6. The hyperbolic boundary (or the Gromov boundary) of ( $S, E)$, denoted by $\partial(S, E)$, is the collection of all equivalence classes according to the relation in equation (3).

2.4. Generalized Ancona theorem. Recall that $\rho(\phi)$ is the radius of convergence of Green's function. Consider a finite-range random walk on a hyperbolic graph. Then, for all $\lambda>\rho(\phi)$, the minimal $\lambda$-Martin boundary of the walk and the hyperbolic boundary of the graph coincide. This was first proven by Series for random walks on Fuchsian groups [47] and then by Ancona for more general hyperbolic graphs [3, 4]. See also [27] for a similar result in more general spaces and [9] for the connection between Ancona's inequality and the Green metric. Later on, this result was proved at the critical value $\lambda=\rho(\phi)$ by Gouëzel and Lalley for random walks on Fuchsian groups [25] and by Gouëzel for symmetric random walks on hyperbolic groups [24].

To prove the main results of $\S \S 4$ and 5, we extend Ancona's theorem to the Ruelle operator setting. In particular, we show that if the potential is uniformly irreducible with respect to a hyperbolic graph (see Definition 2.7) then for all $\lambda>\rho(\phi)$, the minimal Martin boundary $\mathcal{M}_{m}(\lambda)$ and the hyperbolic boundary coincide. The proof of the theorem, which is of technical flavor, appears in Appendix A.

Definition 2.7. Let $(S, E)$ be a connected, undirected and locally finite graph. We say that $\phi$ is uniformly irreducible with respect to $(S, E)$ if:

(1) $\phi$ is bounded;

(2) for every $a, b \in S$ with $[a, b] \neq \varnothing$ we have that $(a, b) \in E$; 
(3) there exists $K>0$ such that for every $a, b \in S$ with $(a, b) \in E$, there exists $k \leq K$ with

$$
L_{\phi}^{k} 1_{[a]}(x)>0 \text { for all } x \in[b] .
$$

Remark 2.8. There may be $(a, b) \in E$ with $[a, b]=\varnothing$. The set of edges $E$ is symmetric and we may have different values of $k$ for $(a, b)$ and $(b, a)$. However, both values are still bounded by $K$.

For every $a \in S$, we pick $x_{a} \in T[a]$ arbitrarily.

THEOREM 2.9. Make the following assumptions.

- $(X, T)$ is locally compact and topologically transitive.

- $\quad \phi$ has summable variations and $P_{G}(\phi)<\infty$.

- There exist $\delta \geq 0$ and a $\delta$-hyperbolic graph $(S, E)$ such that $\phi$ is uniformly irreducible with respect to $(S, E)$.

Then, for every $\lambda>\rho(\phi)$, there is a bijection $\omega: \partial(S, E) \rightarrow \mathcal{M}_{m}(\lambda)$ such that, for any $a_{n} \in S$,

$a_{n} \underset{n \rightarrow \infty}{\longrightarrow} \xi \in \partial(S, E) \Longleftrightarrow$ for all $f \in C_{c}(X), K\left(f, a_{n} x_{a_{n}} \mid \lambda\right) \underset{n \rightarrow \infty}{\longrightarrow} K(f, \omega(\xi) \mid \lambda)$.

Recently and independently, in [8] Bispo and Stadlbauer have managed to show that if $X$ is a group extension of a hyperbolic group and Green's function is quasi-symmetric then the result of Theorem 2.9 also holds at the critical value $\lambda=\rho(\phi)$. In contrast to our proof which is based on [54], their proof is based on the previous works by Lalley and Gouëzel [25] and by Gouëzel [24].

2.5. Regular covers of compact hyperbolic surfaces. Recall that $\mathbb{D}=\{z \in \mathbb{C}:|z|<1\}$ is the unit open hyperbolic disc and that $\partial \mathbb{D}=\{z \in \mathbb{C}:|z|=1\}$ is its boundary. We denote by $d_{\mathbb{D}}$ the hyperbolic metric on $\mathbb{D}$. A Fuchsian group $\Gamma_{0}$ is said to be cocompact if $\mathbb{D} / \Gamma_{0}$ is a compact surface. A regular cover of $\mathbb{D} / \Gamma_{0}$ is a surface $\mathbb{D} / \Gamma$ where $\Gamma \triangleleft \Gamma_{0}$. The group of deck transformations $G$ can be identified with $\Gamma_{0} / \Gamma$ as follows: $\gamma \Gamma \cdot x \Gamma=\gamma x \Gamma$, with $\gamma \in \Gamma_{0}$ and $x \in \mathbb{D}$. Let $\operatorname{Fix}\left(\Gamma_{0}\right)=\left\{\xi \in \partial \mathbb{D}: \exists \gamma_{0} \in \Gamma_{0} \backslash\{\mathrm{id}\}\right.$ such that $\left.\gamma_{0} \xi=\xi\right\}$. Notice that $\operatorname{Fix}\left(\Gamma_{0}\right)$ is a countable set.

Recall that $\delta_{\Gamma}$ is the critical exponent of $\Gamma$, namely the critical value of $\delta$ such that the Poincaré series

$$
\boldsymbol{p}(\Gamma, \delta):=\sum_{\gamma \in \Gamma} e^{-\delta d_{\mathbb{D}}(0, \gamma 0)}
$$

converges for all $\delta>\delta_{\Gamma}$ and diverges for all $\delta<\delta_{\Gamma}$. In general, $\delta_{\Gamma} \leq \delta_{\Gamma_{0}}$ and there is an equality if and only if $G$ is amenable [50]. See also $[15,19,26]$ for similar results in more general spaces. Since $\Gamma_{0}$ is cocompact, $\delta_{\Gamma_{0}}=1$; see [36, Theorem 1.6.3].

We remind the reader of the definition of a conformal measure.

Definition 2.10. (Sullivan [52]) A finite positive measure $\mu$ on $\partial \mathbb{D}$ is said to be $(\Gamma, \delta)$-conformal if for every $\gamma \in \Gamma$,

$$
\frac{d(\mu \circ \gamma)}{d \mu}=\left|\gamma^{\prime}\right|^{\delta}
$$


where $(\mu \circ \gamma)(A)=\mu(\gamma A)=\int 1_{A}\left(\gamma^{-1} x\right) d \mu(x)$. We denote by $\operatorname{Conf}(\Gamma, \delta)$ the collection of all $(\Gamma, \delta)$-conformal measures and by $\operatorname{ext}(\operatorname{Conf}(\Gamma, \delta))$ the extremal points of $\operatorname{Conf}(\Gamma, \delta)$.

Patterson and Sullivan originally considered what they called conformal densities rather than conformal measures. However, both definitions coincide; see [5, Remark 3.3].

2.6. The Bowen-Series coding and its group extension. Let $\Gamma_{0}$ be a cocompact Fuchsian group and let $F_{0} \subseteq \mathbb{D}$ be a fundamental domain of $\mathbb{D} / \Gamma_{0}$ with even corners. In [11], Bowen and Series constructed (with respect to $F_{0}$ ) a finite partition $\left\{I_{a}\right\}_{a \in S_{0}}$ of $\partial \mathbb{D}$ into closed arcs with disjoint interiors, a finite set $\left\{e_{a}\right\}_{a \in S_{0}} \subseteq \Gamma_{0}$ and a map $f_{\Gamma_{0}}: \partial \mathbb{D} \rightarrow \partial \mathbb{D}$ with the following properties.

(Gen) The set $\left\{e_{a}\right\}_{a \in S_{0}}$ is symmetric and generates $\Gamma_{0}$.

(Res) For all $a \in S_{0}, f_{\Gamma_{0}}=e_{a}^{-1}$ on $\operatorname{int}\left(I_{a}\right)$.

(Mar) $\quad\left\{I_{a}\right\}$ is a Markov partition: if $\operatorname{int}\left(f_{\Gamma_{0}}\left(I_{a}\right)\right) \cap \operatorname{int}\left(I_{b}\right) \neq \varnothing$ then $I_{b} \subseteq f\left(I_{a}\right)$.

(Tr) For every $a, b \in S_{0}$ there exists $n$ such that $f_{\Gamma_{0}}^{n}\left(I_{a}\right) \supseteq I_{b}$.

(Orb) For all except finitely many $\xi_{1}, \xi_{2} \in \partial \mathbb{D}$,

there exist $n, m \in \mathbb{N}$ such that $f_{\Gamma_{0}}^{n}\left(\xi_{1}\right)=f_{\Gamma_{0}}^{m}\left(\xi_{2}\right)$

$\Longleftrightarrow$ there exists $\gamma_{0} \in \Gamma_{0}$ such that $\xi_{1}=\gamma_{0}\left(\xi_{2}\right)$.

(Dist) There exists a constant $B>1$ such that for every $a_{1}, \ldots, a_{n} \in S_{0}$ and every $\xi_{1}, \xi_{2} \in \partial \mathbb{D}$ with $f_{\Gamma_{0}}^{k} \xi_{i}=e_{a_{k}}^{-1} \ldots e_{a_{1}}^{-1} \xi_{i}$ for $k=1, \ldots, n$ and $i=1,2$,

$$
\frac{\left|\left(f_{\Gamma_{0}}^{n-1}\right)^{\prime}\left(\xi_{1}\right)\right|}{\left|\left(f_{\Gamma_{0}}^{n-1}\right)^{\prime}\left(\xi_{2}\right)\right|} \leq B .
$$

For (Orb), see [46]. For (Dist) see also [33]. The finiteness of $S_{0}$ follows from the absence of parabolic elements in $\Gamma_{0}$.

We write $I_{a_{1}, \ldots, a_{n}}=\bigcap_{i=1}^{n} f_{\Gamma_{0}}^{-i+1} I_{a_{i}}$. In particular, for all $\xi \in \operatorname{int}\left(I_{a_{1}, \ldots, a_{n}}\right)$ and $1 \leq k \leq n$,

$$
f_{\Gamma_{0}}^{k}(\xi)=e_{a_{k}}^{-1} \cdots e_{a_{1}}^{-1} \xi
$$

For an admissible word $w=\left(a_{1}, \ldots, a_{n}\right)$, we write $e_{w}=e_{a_{1}} \ldots e_{a_{n}}$.

Definition 2.11. A sequence $\left(a_{i}\right)_{i \geq 0}$ with $a_{i} \in S_{0}$ is called a boundary expansion of a point $\xi \in \partial \mathbb{D}$ if for every $n \geq 0, f_{\Gamma_{0}}^{n}(\xi) \in I_{a_{n}}$.

Let

$$
\Sigma=\left\{\left(\sigma_{i}\right)_{i \geq 0}: \forall i \geq 0, \sigma_{i} \in S_{0} \text { and } \operatorname{int}\left(I_{\sigma_{i+1}}\right) \subseteq \operatorname{int}\left(f\left(I_{\sigma_{i}}\right)\right)\right\}
$$

and let $T_{\Sigma}: \Sigma \rightarrow \Sigma$ be the left-shift. Let $\pi_{\Sigma}: \Sigma \rightarrow \partial \mathbb{D}$ be the canonical projection, $\pi_{\Sigma}(\sigma) \in \bigcap_{n \geq 0} \overline{f_{\Gamma_{0}}^{-n} I_{\sigma_{n}}}$ (the intersection is a singleton; see [6]). By (Mar) and (Tr), $(\Sigma, T)$ is a one-sided transitive TMS, and by (Res), $f_{\Gamma_{0}} \circ \pi_{\Sigma}=\pi_{\Sigma} \circ T_{\Sigma}$. Given a point $\sigma \in \Sigma$, we write $\sigma_{i}$ for its $i$ th coordinate. 
Let $G=\Gamma_{0} / \Gamma$ where $\Gamma \triangleleft \Gamma_{0}$. Let $(X, T)$ be the one-sided TMS over the set of states $S_{X}=S_{0} \times G$ with the transition rule

$$
\left(a, \gamma_{1} \Gamma\right) \rightsquigarrow\left(b, \gamma_{2} \Gamma\right) \Longleftrightarrow \operatorname{int}\left(I_{b}\right) \subseteq \operatorname{int}\left(f_{\Gamma_{0}}\left(I_{a}\right)\right) \text { and } \gamma_{2} \Gamma=e_{a}^{-1} \gamma_{1} \Gamma .
$$

The shift space $(X, T)$ is called the group extension, or the G-extension, of $\Sigma$; see [50]. The transitivity of $(X, T)$ follows immediately from the transitivity of the geodesic flow on $\mathbb{D} / \Gamma$ (see [20, Theorem 3.8]) and from the conjugation between cutting sequences and the Bowen-Series coding (see [2]).

We denote by $\pi_{X}: X \rightarrow \Sigma$ the natural projection from $X$ to $\Sigma$.

Definition 2.12. Given $\delta>0$, let $\phi^{\Sigma, \delta}: \Sigma \rightarrow \mathbb{R}$,

$$
\phi^{\Sigma, \delta}(\sigma):=-\delta \log \left|\left(e_{\sigma_{0}}^{-1}\right)^{\prime}\left(\pi_{\Sigma}(\sigma)\right)\right|
$$

and let

$$
\phi^{X, \delta}(x):=\phi^{\Sigma, \delta}\left(\pi_{X}(x)\right) .
$$

The potential $\phi^{\Sigma, \delta}$ is Hölder continuous (see [46]) and thus $\phi^{X, \delta}$ is Hölder continuous as well. It is elementary to show that the potential $\phi^{X, \delta}$ is transient if and only if $\boldsymbol{p}(\Gamma, \delta)<\infty$ and that for all $\delta>\delta_{\Gamma}, P_{G}\left(\phi^{X, \delta}\right)<0$. The following proposition allows us to narrow our discussion to non-atomic measures.

Proposition 2.13. Let $\Gamma_{0}$ be a cocompact Fuchsian group, let $\Gamma \triangleleft \Gamma_{0}$ and let $\delta \geq \delta_{\Gamma}$.

(1) Every $(\Gamma, \delta)$-conformal measure is non-atomic.

(2) Every Radon eigenmeasure of $L_{\phi^{X, \delta}}$ for eigenvalue 1 is non-atomic.

Part (1) of the proposition was proven in [21] for Kleinian groups with no parabolic elements. We prove part (2) in Appendix B.

\section{The eigenmeasures of the Ruelle operator and the conformal measures}

In this section we prove Theorem 1.1, which relates the $(\Gamma, \delta)$-conformal measures to the eigenmeasures of $L_{\phi^{X, \delta}}$ for eigenvalue 1 .

Before proving the theorem, we illustrate the usefulness of Theorem 1.1 and deduce several elementary results, some already known, using the theorem.

COROLlary 3.1. Let $\delta \geq \delta$. Then, for every $\mu \in \operatorname{Conf}(\Gamma, \delta)$, there exists a unique finite measure $v$ on $\operatorname{ext}(\operatorname{Conf}(\Gamma, \delta))$ such that

$$
\mu=\int_{\mu^{\prime} \in \operatorname{ext}(\operatorname{Conf}(\Gamma, \delta))} \mu^{\prime} d v\left(\mu^{\prime}\right)
$$

Proof. This result can be derived from the classical Choquet theory but also follows from the unique representation on the minimal boundary (see equation (2) and also [49]) and by the linearity of the mapping in Theorem 1.1.

Corollary 3.2. (Furstenberg [22]) Assume that $\mathbb{D} / \Gamma$ is compact. Then a $(\Gamma, \delta)$ conformal measure exists if and only if $\delta=\delta_{\Gamma}$. Moreover, the $\left(\Gamma, \delta_{\Gamma}\right)$-conformal measure is unique up to scaling. 
Proof. The corollary follows directly from Ruelle's Perron-Frobenius theorem; see [10].

Corollary 3.3. (Cf. Sullivan [53] and the Hopf-Tsuji-Sullivan theorem.) Assume that $\boldsymbol{p}\left(\Gamma, \delta_{\Gamma}\right)=\infty$. Then the $\left(\Gamma, \delta_{\Gamma}\right)$-conformal measure is unique up to scaling.

Proof. Since $\boldsymbol{p}\left(\Gamma, \delta_{\Gamma}\right)=\infty$, the potential $\phi^{X, \delta_{\Gamma}}$ is recurrent. By Sarig's generalized Ruelle Perron-Frobenius theorem [41, 42], the eigenmeasure of $L_{\phi^{X, \delta_{\Gamma}}}$ is unique up to normalization.

In what follows, let $Y=\partial \mathbb{D} \times G$. The group $\Gamma_{0}$ acts on $Y$ in the following way:

$$
\gamma_{0}(\xi, \gamma \Gamma)=\left(\gamma_{0} \xi, \gamma_{0} \gamma \Gamma\right), \quad \gamma_{0} \in \Gamma_{0},(\xi, \gamma \Gamma) \in Y .
$$

Let $f_{Y}: Y \rightarrow Y$ be the extension of $f_{\Gamma_{0}}$ to $Y$ :

$$
f_{Y}(\xi, \gamma \Gamma)=\left(e_{a}^{-1} \xi, e_{a}^{-1} \gamma \Gamma\right), \quad \xi \in \operatorname{int}\left(I_{a}\right) .
$$

By Proposition 2.13, we can narrow our discussion to non-atomic measures and thus we may ignore the values of $f_{Y}$ on $\partial I_{a}$. To prove Theorem 1.1, we map, in several steps, the Radon eigenmeasures of $L_{\phi}^{X, \delta}$ for eigenvalue 1 to the Radon measures on $Y$ which satisfy $\Gamma_{0}$-regularity condition; see equation (8) in the following lemma.

LEMmA 3.4. Let $\mu_{Y}$ be a non-atomic Radon measure on $Y$. Then the following statements are equivalent.

(1) The measure $\mu_{Y} \circ f_{\Gamma_{0}}$ given by

$$
\left(\mu_{Y} \circ f_{Y}\right)(A \times\{\gamma \Gamma\})=\sum_{a \in S_{0}} \mu_{Y}\left(f_{Y}\left(\left(I_{a} \cap A\right) \times\{\gamma \Gamma\}\right)\right)
$$

with $A \subseteq \partial \mathbb{D}$ measurable is absolutely continuous with respect to $\mu_{Y}$ and

$$
\frac{d\left(\mu_{Y} \circ f_{Y}\right)}{d \mu_{Y}}(\xi, \gamma \Gamma)=\left|f_{\Gamma_{0}}^{\prime}(\xi)\right|^{\delta}, \quad \mu_{Y} \text {-a.e. }
$$

(2) The measure $\mu_{Y}$ is $\Gamma_{0}$-quasi-invariant and for all $\gamma_{0} \in \Gamma_{0}$,

$$
\frac{d\left(\mu_{Y} \circ \gamma_{0}\right)}{d \mu_{Y}}(\xi, \gamma \Gamma)=\left|\gamma_{0}^{\prime}(\xi)\right|^{\delta}, \quad \mu_{Y} \text {-a.e. }
$$

Proof. Assume (1) holds. Fix $\gamma_{0} \in \Gamma_{0}, \gamma_{0} \neq \mathrm{id}_{\Gamma_{0}}$ and let

$$
A_{n, m}=\left\{\xi \in \partial \mathbb{D}: f_{\Gamma_{0}}^{n}(\xi)=\left(f_{\Gamma_{0}}^{m} \circ \gamma_{0}\right)(\xi)\right\} .
$$

By (Orb), $\mu_{Y}\left(\left(\bigcup_{n, m \geq 0} A_{n, m} \times G\right) \triangle Y\right)=0$. Fix $n, m \geq 0$ and let $\xi \in A_{n, m} \backslash \operatorname{Fix}\left(\Gamma_{0}\right)$. Let $a_{1}, \ldots, a_{n}, b_{1}, \ldots, b_{m} \in S_{0}$ such that $\xi \in I_{a_{1}, \ldots, a_{n}}$ and $\gamma_{0} \xi \in I_{b_{1}, \ldots, b_{m}}$. Then

$$
e_{a_{n}}^{-1} \cdots e_{a_{1}}^{-1}(\xi)=e_{b_{m}}^{-1} \cdots e_{b_{1}}^{-1} \gamma_{0}(\xi)
$$

In particular, $\xi$ is a fixed point of $e_{a_{1}} \cdots e_{a_{n}} e_{b_{m}}^{-1} \cdots e_{b_{1}}^{-1} \gamma_{0}$. Since $\xi \notin \operatorname{Fix}\left(\Gamma_{0}\right)$,

$$
\gamma_{0}=\left(e_{b_{m}}^{-1} \cdots e_{b_{1}}^{-1}\right)^{-1} e_{a_{n}}^{-1} \cdots e_{a_{1}}^{-1}
$$


and

$$
\begin{aligned}
\left|\gamma_{0}^{\prime}(\xi)\right|^{\delta} & =\left|\left(\left(e_{b_{m}}^{-1} \cdots e_{b_{1}}^{-1}\right)^{-1}\right)^{\prime}\left(e_{a_{n}}^{-1} \cdots e_{a_{2}}^{-1} \xi\right)\right|^{\delta} \cdot\left|\left(e_{a_{n}}^{-1} \cdots e_{a_{1}}^{-1}\right)^{\prime}(\xi)\right|^{\delta} \\
& =\frac{\left|\left(e_{a_{n}}^{-1} \cdots e_{a_{1}}^{-1}\right)^{\prime}(\xi)\right|^{\delta}}{\left|\left(e_{b_{m}}^{-1} \cdots e_{b_{1}}^{-1}\right)^{\prime}\left(\gamma_{0} \xi\right)\right|^{\delta}} .
\end{aligned}
$$

For $\mu_{Y}$-a.e. $(\xi, \gamma \Gamma) \in\left(I_{a_{1}, \ldots, a_{n}} \cap \gamma_{0}^{-1} I_{b_{1}, \ldots, b_{m}}\right) \times G$ we have that

$$
\begin{aligned}
\frac{d\left(\mu_{Y} \circ f_{Y}^{n}\right)}{d \mu_{Y}}(\xi, \gamma \Gamma) & =\frac{d\left(\mu_{Y} \circ f_{Y}^{m} \circ \gamma_{0}\right)}{d \mu_{Y}}(\xi, \gamma \Gamma) \\
& =\frac{d\left(\mu_{Y} \circ f_{Y}^{m}\right)}{d \mu_{Y}}\left(\gamma_{0} \xi, \gamma_{0} \gamma \Gamma\right) \frac{d\left(\mu_{Y} \circ \gamma_{0}\right)}{d \mu_{Y}}(\xi, \gamma \Gamma) .
\end{aligned}
$$

Moreover, by equation (7), for $\mu_{Y}$-a.e. $(\xi, \gamma \Gamma) \in\left(I_{a_{1}, \ldots, a_{n}} \cap \gamma_{0}^{-1} I_{b_{1}, \ldots, b_{m}}\right) \times G$,

$$
\frac{d\left(\mu_{Y} \circ f_{Y}^{n}\right)}{d \mu_{Y}}(\xi, \gamma \Gamma)=\left|\left(e_{a_{n}}^{-1} \cdots e_{a_{1}}^{-1}\right)^{\prime}(\xi)\right|^{\delta}
$$

and

$$
\frac{d\left(\mu_{Y} \circ f_{Y}^{m}\right)}{d \mu_{Y}}\left(\gamma_{0} \xi, \gamma_{0} \gamma \Gamma\right)=\left|\left(e_{b_{m}}^{-1} \cdots e_{b_{1}}^{-1}\right)^{\prime}\left(\gamma_{0} \xi\right)\right|^{\delta} .
$$

Thus, for $\mu_{Y}$-a.e. $(\xi, \gamma \Gamma) \in\left(I_{a_{1}, \ldots, a_{n}} \cap \gamma_{0}^{-1} I_{b_{1}, \ldots, b_{m}}\right) \times G$,

$$
\frac{d\left(\mu_{Y} \circ \gamma_{0}\right)}{d \mu_{Y}}(\xi, \gamma \Gamma)=\left(\frac{d\left(\mu_{Y} \circ f_{Y}^{m}\right)}{d \mu_{Y}}\left(\gamma_{0} \xi, \gamma_{0} \gamma \Gamma\right)\right)^{-1} \frac{d\left(\mu_{Y} \circ f_{Y}^{n}\right)}{d \mu_{Y}}(\xi, \gamma \Gamma)=\left|\gamma_{0}^{\prime}(\xi)\right|^{\delta} \text {. }
$$

Since there are only a countable number of such $\left(a_{1}, \ldots, a_{n}\right),\left(b_{1}, \ldots, b_{m}\right), m$ and $n$, the identity holds for $\mu_{Y}$-a.e. $(\xi, \gamma \Gamma) \in Y$. So $(1) \Rightarrow(2)$.

Next, assume (2). Fix $a \in S_{0}$. Then, for $\mu_{Y}$-a.e. $(\xi, \gamma \Gamma) \in I_{a} \times G$,

$$
\frac{d\left(\mu_{Y} \circ f_{Y}\right)}{d \mu_{Y}}(\xi, \gamma \Gamma)=\frac{d\left(\mu_{Y} \circ e_{a}^{-1}\right)}{d \mu_{Y}}(\xi, \gamma \Gamma)=\left|\left(e_{a}^{-1}\right)^{\prime}(\xi)\right|^{\delta}=\left|f_{\Gamma_{0}}^{\prime}(\xi)\right|^{\delta} .
$$

Henceforth we use the following canonical correspondence to identify $X$ with $\Sigma \times G$ :

$$
(\sigma, \gamma \Gamma) \longmapsto\left(\left(\sigma_{0}, \gamma \Gamma\right),\left(\sigma_{1}, e_{\sigma_{0}}^{-1} \gamma \Gamma\right),\left(\sigma_{1}, e_{\sigma_{1}}^{-1} \gamma \Gamma\right), \ldots\right) .
$$

In particular, we will not distinguish between the two. We let $\tilde{\pi}: X \rightarrow Y, \tilde{\pi}(\sigma, \gamma \Gamma)=$ $\left(\pi_{\Sigma}(\sigma), \gamma \Gamma\right)$.

LEMMA 3.5. Let $\delta \geq \delta_{\Gamma}$. Then the map $\mu_{X} \mapsto \mu_{X} \circ \tilde{\pi}^{-1}$ is an affine bijection between the Radon eigenmeasures of $L_{\phi^{X, \delta}}$ with eigenvalue 1 and the non-atomic Radon measures on $Y$ which satisfy equation (8).

Proof. Recall that $\pi_{\Sigma}$ is bijective away from a countable number of points; see [46]. Therefore, since all eigenmeasures of the Ruelle operator are non-atomic (see Proposition 2.13), $\tilde{\pi}$ is a measure-theoretic isomorphism.

Recall that $\mu_{X}$ is an eigenmeasure of $L_{\phi^{X, \delta}}$ of eigenvalue 1 if and only if

$$
\frac{d\left(\mu_{X} \circ T\right)}{d \mu_{X}}(\sigma, \gamma \Gamma)=\left|f_{\Gamma_{0}}^{\prime}(\pi(\sigma))\right|^{\delta}
$$


where $\left(\mu_{X} \circ T\right)(A \times\{\gamma \Gamma\})=\sum_{a \in S_{0}} \mu_{X}(T(([a] \cap A) \times\{\gamma \Gamma\}))$; see [44] and references therein. Since $\tilde{\pi} \circ f_{Y}=T \circ \tilde{\pi}$,

$$
\begin{aligned}
\frac{d\left(\mu_{Y} \circ f_{Y}\right)}{d \mu_{Y}}\left(\left(\pi^{-1}(\sigma), \gamma \Gamma\right)\right) & =\frac{d\left(\mu_{Y} \circ\left(f_{Y} \circ \tilde{\pi}^{-1}\right)\right)}{d\left(\mu_{Y} \circ \tilde{\pi}^{-1}\right)}(\sigma, \gamma \Gamma) \\
& =\frac{d\left(\mu_{X} \circ T\right)}{d \mu_{X}}(\sigma, \gamma \Gamma) \\
& =\left|f_{\Gamma_{0}}^{\prime}(\pi(\sigma))\right|^{\delta} .
\end{aligned}
$$

Hence, by Lemma 3.4, $\mu_{X}$ is an eigenmeasure if and only if $\mu_{Y}$ satisfies equation (8).

Proof of Theorem 1.1. By Lemma 3.5, it suffices to present a bijection between the $(\Gamma, \delta)$-conformal measures and the non-atomic Radon measures on $Y$ which satisfy equation (8).

Let $\mu \in \operatorname{Conf}(\Gamma, \delta)$. We define a new measure $\mu_{Y}$ on $Y=\partial \mathbb{D} \times G$ as follows: for $A \subseteq$ $\partial \mathbb{D}$ and $\gamma \Gamma \in G$,

$$
\mu_{Y}(A \times\{\gamma \Gamma\}):=\int\left|\gamma^{\prime}(\xi)\right|^{\delta} 1_{A}(\gamma \xi) d \mu(\xi) .
$$

We show that this definition does not depend on the choice of $\gamma$ which represents $\gamma \Gamma$. Assume that $\gamma_{1} \Gamma=\gamma_{2} \Gamma$ and let $\gamma \in \Gamma$ such that $\gamma_{1}=\gamma_{2} \gamma$. Since $\mu$ is $(\Gamma, \delta)$-conformal,

$$
\begin{aligned}
\int\left|\gamma_{1}^{\prime}(\xi)\right|^{\delta} 1_{A}\left(\gamma_{1} \xi\right) d \mu(\xi) & =\int\left|\left(\gamma_{2} \gamma\right)^{\prime}(\xi)\right|^{\delta} 1_{A}\left(\gamma_{2} \gamma \xi\right) d \mu(\xi) \\
& =\int\left|\gamma_{2}^{\prime}(\gamma \xi)\right|^{\delta}\left|\gamma^{\prime}(\xi)\right|^{\delta} 1_{A}\left(\gamma_{2} \gamma \xi\right) d \mu(\xi) \\
& =\int\left|\gamma_{2}^{\prime}(\xi)\right|^{\delta}\left|\gamma^{\prime}\left(\gamma^{-1} \xi\right)\right|^{\delta} 1_{A}\left(\gamma_{2} \xi\right) \frac{d\left(\mu \circ \gamma^{-1}\right)}{d \mu}(\xi) d \mu(\xi) \\
& =\int\left|\gamma_{2}^{\prime}(\xi)\right|^{\delta} 1_{A}\left(\gamma_{2} \xi\right) d \mu(\xi) .
\end{aligned}
$$

So $\mu_{Y}$ is defined properly. Since $\mu$ is non-atomic (see Proposition 2.13), $\mu_{Y}$ is non-atomic. Moreover, by definition different choices of $\mu$ lead to different measures $\mu_{Y}$ (consider $\gamma \in \Gamma)$.

We prove that $\mu_{Y}$ satisfies equation (8). Given $A \subseteq \partial \mathbb{D}$ Borel and $\gamma_{1}, \gamma_{2} \in \Gamma_{0}$,

$$
\begin{aligned}
\left(\mu_{Y} \circ \gamma_{1}\right)\left(A \times\left\{\gamma_{2} \Gamma\right\}\right) & =\left(\mu_{Y}\right)\left(\gamma_{1} A \times\left\{\gamma_{1} \gamma_{2} \Gamma\right\}\right) \\
& =\int\left|\left(\gamma_{1} \gamma_{2}\right)^{\prime}(\xi)\right|^{\delta} 1_{\gamma_{1} A}\left(\gamma_{1} \gamma_{2} \xi\right) d \mu(\xi) \\
& =\int\left|\left(\gamma_{1} \gamma_{2}\right)^{\prime}(\xi)\right|^{\delta} 1_{A}\left(\gamma_{2} \xi\right) d \mu(\xi) \\
& =\int\left|\left(\gamma_{1}\right)^{\prime}\left(\gamma_{2} \xi\right)\right|^{\delta}\left|\left(\gamma_{2}\right)^{\prime}(\xi)\right|^{\delta} 1_{A}\left(\gamma_{2} \xi\right) d \mu(\xi) .
\end{aligned}
$$

By the definition of $\mu_{Y}$,

$$
\mu_{Y}\left(\left|\gamma_{1}^{\prime}\right|^{\delta} 1_{A \times\left\{\gamma_{2} \Gamma\right\}}\right)=\int\left|\left(\gamma_{1}\right)^{\prime}\left(\gamma_{2} \xi\right)\right|^{\delta}\left|\left(\gamma_{2}\right)^{\prime}(\xi)\right|^{\delta} 1_{A}\left(\gamma_{2} \xi\right) d \mu(\xi)
$$

and therefore $d\left(\mu_{Y} \circ \gamma_{1}\right) / d \mu_{Y}=\left|\gamma_{1}^{\prime}\right|^{\delta}$ for all $\gamma_{1} \in \Gamma_{0}$. 
Finally, we show that this mapping is onto. Given a non-atomic Radon measure $\mu_{Y}$ which satisfies equation (8), let $\mu(\cdot):=\mu_{Y}(\cdot,\{\Gamma\})$. Clearly $\mu$ is non-atomic and $\mu_{Y}$ is the resulting measure of the mapping in equation (9). Moreover, for every $\gamma \in \Gamma$,

$$
\begin{aligned}
(\mu \circ \gamma)(A) & =\mu_{Y}(\gamma A,\{\Gamma\}) \\
& =\mu_{Y}(\gamma A,\{\gamma \Gamma\}) \quad\left(\because \gamma \Gamma=\Gamma \text { in } G=\Gamma_{0} / \Gamma\right) \\
& =\left(\mu_{Y} \circ \gamma\right)(A,\{\Gamma\}) \\
& =\mu_{Y}\left(\left|\gamma^{\prime}\right|^{\delta} 1_{A \times\{\Gamma\}}\right) \\
& =\int\left|\gamma^{\prime}(\xi)\right|^{\delta} 1_{A}(\xi) \mu(\xi)
\end{aligned}
$$

and $\mu$ is indeed a $(\Gamma, \delta)$-conformal measure.

\section{Application to hyperbolic covers}

We now turn our attention to study regular covers with a hyperbolic group of deck transformations. Recall that $G$ is called a hyperbolic group if some (or every, see [23]) Cayley graph of $G$ is a hyperbolic graph. We denote by $\partial G$ the hyperbolic boundary of $G$; see $\S 2.3$. We say that a regular cover $\mathbb{D} / \Gamma$ of $\mathbb{D} / \Gamma_{0}$ is a hyperbolic cover if the group of deck transformations $G=\Gamma_{0} / \Gamma$ is a hyperbolic group.

In this section we prove Theorem 1.2, which describes the extremal conformal measures of $\Gamma$ in terms of $\partial G$. In principle, we would like to apply the generalized Ancona theorem on the Markov shift $X$ and the potential $\phi^{X, \delta}$. Since we do not know a priori that the states graph of $X$ is undirected or hyperbolic, we work with a 'larger' hyperbolic graph structure on $S_{X}=S_{0} \times G$ which is easier to analyze. In what follows, let

$$
E_{G}=\left\{\left(g_{1}, g_{2}\right) \in G \times G: g_{1}=g_{2} \text { or } \exists a \in S_{0} \text { such that } e_{a}^{-1} g_{1}=g_{2}\right\}
$$

and let

$$
E_{X}=\left\{((a, g),(b, h)) \in S_{X} \times S_{X}:(g, h) \in E_{G}\right\} .
$$

Since $\left\{e_{a}\right\}_{a \in S_{0}}$ is a symmetric set which generates $\Gamma_{0}$, the set $\left\{e_{a} \Gamma\right\}_{a \in S_{0}}$ generates $G$ and $\left(G, E_{G}\right)$ is an undirected Cayley graph of $G$. Since $\left(G, E_{G}\right)$ is undirected, $\left(S_{X}, E_{X}\right)$ is undirected as well. Let $\pi_{S_{X}}: S_{X} \rightarrow G$ be the natural projection, $\pi_{S_{X}}(\xi, g)=g$. Observe that $\left(S_{X}, E_{X}\right)$ is not the canonical graph associated to the transition matrix of the TMS $X$. In fact, it is larger since it has more edges.

Definition 4.1. Two metric spaces $\left(X_{1}, d_{1}\right)$ and $\left(X_{2}, d_{2}\right)$ are called quasi-isometric if there exist $g: X_{1} \rightarrow X_{2}, A \geq 1, B \geq 0$ and $C \geq 0$ such that the following statements hold.

(1) For every $x, y \in X_{1}$,

$$
\frac{1}{A} d_{1}(x, y)-B \leq d_{2}(g(x), g(y)) \leq A d_{1}(x, y)+B .
$$

(2) For every $y \in X_{2}$ there exists $x \in X_{1}$ such that

$$
d_{2}(y, g(x)) \leq C .
$$

We call such a function $g$ a quasi-isometry; see [17]. 
Since, for all $a, b \in S_{0}$ and $g, h \in G$,

$$
d_{E_{G}}(g, h) \leq d_{E_{X}}((a, g),(b, h)) \leq d_{E_{G}}(g, h)+1
$$

the graphs $\left(G, E_{G}\right)$ and $\left(S_{X}, E_{X}\right)$ are quasi-isometric. Since hyperbolicity is preserved under quasi-isometries (see [23]), $G$ is a hyperbolic group if and only if $\left(S_{X}, E_{X}\right)$ is a hyperbolic graph. We denote by $\partial G$ and $\partial S_{X}$ the hyperbolic boundaries of $\left(G, E_{G}\right)$ and $\left(S_{X}, E_{X}\right)$, respectively; see the definitions in $\S 2.3$. We fix origin points $o_{G} \in G, o_{S_{X}} \in S_{X}$ with $\pi_{S_{X}}\left(o_{S_{X}}\right)=o_{G}$. Notice that equation (10) together with the definition of $\wedge($ see $\S 2.3)$ implies that

$$
|(a, g) \wedge(b, h)|_{o_{S_{X}}}-1 \leq|g \wedge h|_{o_{G}} \leq|(a, g) \wedge(b, h)|_{o_{S_{X}}}+1 .
$$

In particular, for every $\left\{a_{n}\right\} \subseteq S_{0}$ and $\left\{g_{n}\right\} \subseteq G$,

$$
\lim _{m, n \rightarrow \infty}\left|g_{n} \wedge g_{m}\right|_{o_{G}}=\infty \text { if and only if } \lim _{m, n \rightarrow \infty}\left|\left(a_{n}, g_{n}\right) \wedge\left(a_{m}, g_{m}\right)\right|_{o_{S_{X}}} \rightarrow \infty
$$

and $\left(a_{n}, g_{n}\right)$ converges to a point in $\partial S_{X}$ if and only if $g_{n}=\pi_{S_{X}}\left(a_{n}, g_{n}\right)$ converges to a point in $\partial G$. For $\eta \in \partial S_{X}$, set

$$
\pi_{S_{X}}(\eta):=\lim _{n \rightarrow \infty} g_{n}
$$

where $\left(a_{n}, g_{n}\right) \rightarrow \eta$. It is easy to verify that $\pi_{S_{X}}: \partial S_{X} \rightarrow \partial G$ is a well-defined bijection between the two boundaries.

Recall the definition of uniform irreducibility (see Definition 2.7).

Proposition 4.2. $\phi^{X, \delta}$ is uniformly irreducible with respect to $\left(S_{X}, E_{X}\right)$.

Proof. Since $\Sigma$ is compact, $\phi^{X, \delta}$ is bounded. If $[(a, g),(b, h)] \neq \varnothing$ then $h=e_{a}^{-1} g$. In particular, $(g, h) \in E_{G}$ and thus $((a, g),(b, h)) \in E_{X}$. For every $a, b \in S_{0}$ and $\gamma \in \Gamma_{0}$, let $n_{a, b, \gamma}$ be an integer such that there is an admissible path from $(a, \Gamma)$ to $(b, \gamma \Gamma)$ in $X$, namely

$$
\left(L_{\phi^{X, \delta}}^{n_{a, b, \gamma}}\left(1_{[(a, \Gamma)]}\right)\right)\left(x_{(b, \gamma \Gamma)}\right)>0
$$

where $x_{(b, \gamma \Gamma)} \in T[(b, \gamma \Gamma)]$. Since $(X, T)$ is topologically transitive, such a path exists. Let $((a, g),(b, h)) \in E_{X}$. Then either $g=h$ and

$$
L_{\phi^{X, \delta}}^{n_{a, b, \mathrm{id}}} \Gamma_{0}\left(1_{[(a, g)]}\right)\left(x_{(b, h)}\right)>0
$$

or $h=e_{c}^{-1} g$, for some $c \in S_{0}$, and

$$
L_{\phi^{X, \delta}}^{n_{a, b, e_{c}^{-1}}}\left(1_{[(a, g)]}\right)\left(x_{(b, h)}\right)>0 .
$$

Thus, with

$$
K=\max _{a, b \in S_{0}} \max _{\gamma \in\left\{e_{c}\right\}_{c \in S_{0}} \cup\left\{\mathrm{id}_{\Gamma_{0}}\right\}} n_{a, b, \gamma}
$$

we have that $\phi^{X, \delta}$ is uniformly irreducible with respect to $\left(S_{X}, E_{X}\right)$.

We are now ready to prove the main result of this section. 
Proof of Theorem 1.2. Since $\delta>\delta_{\Gamma}$, we have that $P_{G}\left(\phi^{X, \delta}\right)<0$. By Proposition $4.2, \phi^{X, \delta}$ is uniformly irreducible with respect to the (larger) hyperbolic graph $\left(S_{X}, E_{X}\right)$. Thus the conditions of Theorem 2.9 hold.

Let $\mu \in \operatorname{Conf}(\Gamma, \delta)$. By Corollary 3.1, we can assume without loss of generality that $\mu \in \operatorname{ext}(\operatorname{Conf}(\Gamma, \delta))$. Let $\mu_{X}$ be the corresponding eigenmeasure on $X$ from Theorem 1.1. Since $\mu$ is extremal and the transformation from $\mu$ to $\mu_{X}$ is linear, $\mu_{X}$ is extremal as well. By Theorem 2.9 there exists $\eta^{\prime} \in \partial S_{X}$ such that for $\mu_{X}$-a.e. $x=(\sigma, \Gamma) \in X, T^{n} x \rightarrow \eta^{\prime}$. Let $\eta=\pi_{S_{X}}\left(\eta^{\prime}\right) \in \partial G$. By equation (12) we have that

$$
e_{\sigma_{n}}^{-1} \cdots e_{\sigma_{0}}^{-1} \Gamma \rightarrow \eta
$$

on the Cayley graph $\left(G, E_{G}\right)$. Since $\mu(\cdot)=\mu_{X}\left(\pi_{\Sigma}^{-1}(\cdot) \times\{\Gamma\}\right),(\sigma, \Gamma)$ is $\mu_{X}$-typical point if and only if $\sigma$ is a $\mu$-typical point and thus the first part of the theorem follows.

Now, let $\eta \in \partial G$ and let $\eta^{\prime}=\pi_{S_{X}}^{-1} \eta$. By Theorem 2.9 there is a unique eigenmeasure $\mu_{X}$ such that for $\mu_{X}$-a.e. $x \in X, T^{n} x \rightarrow \eta^{\prime}$. Then, the second part of the theorem follows with $\mu(\cdot)=\mu_{X}\left(\pi_{\Sigma}^{-1}(\cdot) \times\{\Gamma\}\right)$.

\section{Convergence of cutting sequences along geodesics}

In this section we study the asymptotic behavior of cutting sequences on hyperbolic covers with respect to conformal measures. In particular, for every $\delta>\delta_{\Gamma}$ and every $\mu \in \operatorname{Conf}(\Gamma, \delta)$, we show that the cutting sequence (projected to $G$ ) $\mu$-almost surely converges to a point in $\partial G$ (see Theorem 1.3).

We emphasize that the geodesics on a regular cover do not always escape to infinity. In fact, by the Hopf-Tsuji-Sullivan theorem the geodesic flow is conservative with respect to the Liouville measure if and only if the Poincaré series diverges for $\delta=1$; see [1]. An example is a $\mathbb{Z}^{d}$-cover: the Poincaré series diverges if and only if $d \leq 2$ [38].

In what follows, recall that $F_{0} \subseteq \mathbb{D}$ is a fundamental domain of $\mathbb{D} / \Gamma_{0}$ with even corners, that given $\xi^{-}, \xi^{+} \in \partial \mathbb{D}$ with $\xi^{-} \neq \xi^{+}$we denote by $\xi^{-} \wedge \xi^{+}$the unique geodesic curve in $\mathbb{D}$ from $\xi^{-}$to $\xi^{+}$and that

$$
\mathcal{R}=\left\{\left(\xi^{-}, \xi^{+}\right) \in \partial \mathbb{D}^{2}:\left(\xi^{-} \wedge \xi^{+}\right) \cap \operatorname{int}\left(F_{0}\right) \neq \varnothing\right\} .
$$

Observe that $\mathcal{R}$ is symmetric, namely $\left(\xi^{-}, \xi^{+}\right) \in \mathcal{R}$ if and only if $\left(\xi^{+}, \xi^{-}\right) \in \mathcal{R}$. Let $\left(\xi^{-}, \xi^{+}\right) \in \mathcal{R}$ and let $\left\{F_{i}\right\}_{i \in \mathbb{Z}}$ be the sequence of copies of $F_{0}$ that the curve $\left(\xi^{-} \wedge \xi^{+}\right)$ intersects. If $\left(\xi^{-} \wedge \xi^{+}\right)$passes through a vertex of some $F_{i}$, we perturb the curve around it; see [48, Figure 5]. Then, for all $i$, there exists a unique $e_{i} \in\left\{e_{a}\right\}_{a \in S_{0}}$ such that $F_{i}=e_{i}^{-1} F_{i+1}$.

Definition 5.1. The sequence $\left(\ldots, e_{-1}, e_{0}, e_{1}, \ldots\right)$ is called the cutting sequence of $\left(\xi^{-}, \xi^{+}\right)$.

For $\left(\xi^{-}, \xi^{+}\right) \in \mathcal{R}$ with a cutting sequence $\left(\ldots, e_{-1}, e_{0}, e_{1}, \ldots\right)$, recall that

$$
\eta_{n}^{+}\left(\xi^{-}, \xi^{+}\right)=e_{n}^{-1} \cdots e_{0}^{-1} \Gamma
$$

and

$$
\eta_{n}^{-}\left(\xi^{-}, \xi^{+}\right)=\left(e_{-n-1}\right)^{-1} \cdots\left(e_{-1}\right)^{-1} \Gamma
$$


To prove Theorem 1.3, we exploit the connection between boundary expansions and cutting sequences, presented by Series [48] and also by Adler and Flatto [2]. Firstly, we briefly introduce the two-sided Bowen-Series coding. We denote by $\Sigma^{ \pm}$the negative one-sided shift,

$$
\Sigma^{ \pm}=\left\{\left(\ldots, \sigma_{-1}, \sigma_{0}, \sigma_{1}, \ldots\right): \forall i,\left[\sigma_{i}, \sigma_{i+1}\right] \neq \varnothing \text { in } \Sigma\right\} .
$$

We write $T_{\Sigma}$ for the left-shift action on both $\Sigma$ and $\Sigma^{ \pm}$; the meaning should be clear from the context. Recall that $\pi_{\Sigma}: \Sigma \rightarrow \partial \mathbb{D}$ is the canonical projection where

$$
\pi_{\Sigma}\left(\sigma_{0}, \sigma_{1}, \ldots\right) \in \bigcap_{n \geq 0} \overline{f_{\Gamma_{0}}^{-n} I_{\sigma_{n}}} .
$$

We write $\pi^{+}: \Sigma^{ \pm} \rightarrow \partial \mathbb{D}$

$$
\pi^{+}(\sigma)=\pi_{\Sigma}\left(\sigma_{0}, \sigma_{1}, \ldots\right) .
$$

For every $a \in S_{0}$, let $\bar{a} \in S_{0}$ such that $e_{\bar{a}}=e_{a}^{-1}$ and $[a, b] \neq \varnothing$ if and only if $[\bar{b}, \bar{a}] \neq \varnothing$ in $\Sigma$. We define $\pi^{-}(\sigma)=\pi_{\Sigma}\left(\overline{\sigma_{-1}}, \overline{\sigma_{-2}}, \ldots\right)$ and $\pi(\sigma)=\left(\pi^{-}(\sigma), \pi^{+}(\sigma)\right)$. Then

$$
\pi\left(T_{\Sigma} \sigma\right)=\left(\pi^{-}\left(T_{\Sigma} \sigma\right), \pi^{+}\left(T_{\Sigma} \sigma\right)\right)=\left(e_{\sigma_{0}}^{-1} \pi^{-}(\sigma), e_{\sigma_{0}}^{-1} \pi^{+}(\sigma)\right) .
$$

Let

$$
\mathcal{A}=\left\{\left(\xi^{-}, \xi^{+}\right): \exists \sigma \in \Sigma^{ \pm} \text {such that }\left(\xi^{-}, \xi^{+}\right)=\pi(\sigma)\right\} .
$$

The Bowen-Series map $f_{\Gamma_{0}}$ acts on $\mathcal{A}$ similarly to the left-shift action,

$$
f_{\Gamma_{0}}\left(\xi^{-}, \xi^{+}\right)=\left(\pi \circ T_{\Sigma}\right)(\sigma)=\left(e_{\sigma_{0}}^{-1} \pi^{-}(\sigma), e_{\sigma_{0}}^{-1} \pi^{+}(\sigma)\right)=e_{\sigma_{0}}^{-1}\left(\xi^{-}, \xi^{+}\right) .
$$

Here and throughout, $e_{\sigma_{0}}^{-1}\left(\xi^{-}, \xi^{+}\right):=\left(e_{\sigma_{0}}^{-1}\left(\xi^{-}\right), e_{\sigma_{0}}^{-1}\left(\xi^{+}\right)\right)$. The value of the first-return map $g_{\Gamma_{0}}: \mathcal{R} \rightarrow \mathcal{R}$ on a pair $\left(\xi^{-}, \xi^{+}\right) \in \mathcal{R}$ with cutting sequence $\left(\ldots, e_{-1}, e_{0}, e_{1}, \ldots\right)$ is

$$
g_{\Gamma_{0}}\left(\xi^{-}, \xi^{+}\right)=e_{0}^{-1}\left(\xi^{-}, \xi^{+}\right)
$$

Notice that $g_{\Gamma_{0}}\left(\xi^{-}, \xi^{+}\right) \in \mathcal{R}$ and the cutting sequence of $g_{\Gamma_{0}}\left(\xi^{-}, \xi^{+}\right)$is the cutting sequence of $\left(\xi^{-}, \xi^{+}\right)$shifted by one position to the left. In particular,

$$
g_{\Gamma_{0}}^{n}\left(\xi^{-}, \xi^{+}\right)=\left(e_{n-1}^{-1} \cdots e_{0}^{-1}\right)\left(\xi^{-}, \xi^{+}\right) .
$$

THEOREM 5.2. (Series [48]) There exists a bijection $\varphi: \mathcal{A} \rightarrow \mathcal{R}$ such that

$$
\left(\varphi \circ f_{\Gamma_{0}}\right)\left(\xi^{-}, \xi^{+}\right)=\left(g_{\Gamma_{0}} \circ \varphi\right)\left(\xi^{-}, \xi^{+}\right) .
$$

An explicit formula for $\varphi$ was derived in [2].

Let

$$
\mathcal{D}:=\left\{\xi \in \partial \mathbb{D} \backslash \operatorname{Fix}\left(\Gamma_{0}\right):\left|\pi_{\Sigma}^{-1}(\xi)\right|=1\right\} .
$$

Notice that $\mathcal{D}$ is a $\Gamma_{0}$-invariant set and that $\partial \mathbb{D} \backslash \mathcal{D}$ is countable and thus a null set with respect to any conformal measure; see Proposition 2.13. Given $\xi \in \mathcal{D}$ with a (one-sided) boundary expansion $\pi_{\Sigma}^{-1}(\xi)=\left(\sigma_{0}, \sigma_{1} \ldots\right)$ and $\gamma_{0} \in \Gamma_{0}$, we write

$$
\tau_{n}\left(\xi, \gamma_{0}\right):=e_{\sigma_{n}}^{-1} \cdots e_{\sigma_{0}}^{-1} \gamma_{0} \Gamma \text {. }
$$


Proof of Theorem 1.3. We show that for $\mu$-a.e. $\xi^{+} \in \partial \mathbb{D}$, for every $\xi^{-} \in \mathcal{D}$ with $\left(\xi^{-}, \xi^{+}\right) \in \mathcal{R}, \eta_{n}^{+}\left(\xi^{-}, \xi^{+}\right)$converges to a point in $\partial G$. Observe that if the cutting sequence of $\left(\xi^{-}, \xi^{+}\right)$is $\left(e_{i}\right)$ then the cutting sequence of $\left(\xi^{+}, \xi^{-}\right)$is $\left(f_{i}\right)$ with $f_{i}=e_{-i-1}$. In particular,

$$
\eta_{n}^{+}\left(\xi^{+}, \xi^{-}\right)=\left(f_{n}\right)^{-1} \cdots\left(f_{0}\right)^{-1} \Gamma=\left(e_{-n-1}\right)^{-1} \cdots\left(e_{-1}\right)^{-1} \Gamma=\eta_{n}^{-}\left(\xi^{-}, \xi^{+}\right)
$$

and so we prove the theorem only for $\eta_{n}^{+}\left(\xi^{-}, \xi^{+}\right)$as the arguments for $\eta_{n}^{-}\left(\xi^{-}, \xi^{+}\right)$are similar. By Corollary 3.1, we can assume without loss of generality that $\mu^{+}$is extremal. Since $\mu$ is non-atomic (see Proposition 2.13), we can also assume that $\xi^{-}, \xi^{+} \in \mathcal{D}$.

Let $\gamma_{0} \in \Gamma_{0}$, let $\eta \in \partial G$ and let

$$
A_{\gamma_{0}}=\left\{\xi^{+} \in \mathcal{D}: \begin{array}{c}
\exists \xi^{-} \in \mathcal{D} \text { such that }\left(\xi^{-}, \xi^{+}\right) \in \mathcal{R}, \varphi^{-1}\left(\xi^{-}, \xi^{+}\right)=\gamma_{0}\left(\xi^{-}, \xi^{+}\right) \\
\text {and } \lim _{n \rightarrow \infty} \eta_{n}^{+}\left(\xi^{-}, \xi^{+}\right) \neq \eta
\end{array}\right\} .
$$

We write $\lim _{n \rightarrow \infty} \eta_{n}^{+}\left(\xi^{-}, \xi^{+}\right) \neq \eta$ whenever the limit does not exist or it exists but differs from $\eta$. We show that there exists $\eta \in \partial G$ such that $\mu\left(A_{\gamma_{0}}\right)=0$.

Let $\xi^{+} \in A_{\gamma_{0}}$ and let $\xi^{-} \in \mathcal{D}$ such that $\left(\xi^{-}, \xi^{+}\right) \in \mathcal{R}$ and $\varphi^{-1}\left(\xi^{-}, \xi^{+}\right)=\gamma_{0}\left(\xi^{-}, \xi^{+}\right)$. Let $\sigma \in \Sigma$ such that $\pi_{\Sigma}(\sigma)=\gamma_{0} \xi^{+}$and let $\left(\ldots, e_{-1}, e_{0}, e_{1}, \ldots\right)$ be the cutting sequence of $\left(\xi^{-}, \xi^{+}\right)$. For every $n \geq 1$, let $\gamma_{n} \in \Gamma_{0}$ such that $\varphi^{-1}\left(g_{\Gamma_{0}}^{n}\left(\xi^{-}, \xi^{+}\right)\right)=\gamma_{n}\left(g_{\Gamma_{0}}^{n}\left(\xi^{-}, \xi^{+}\right)\right)$. By Theorem 5.2,

$$
\left(\varphi \circ f_{\Gamma_{0}}^{n} \circ \varphi^{-1}\right)\left(\xi^{-}, \xi^{+}\right)=g_{\Gamma_{0}}^{n}\left(\xi^{-}, \xi^{+}\right),
$$

meaning

$$
\left(\gamma_{n}^{-1} e_{\sigma_{n-1}}^{-1} \cdots e_{\sigma_{0}}^{-1} \gamma_{0}\right)\left(\xi^{-}, \xi^{+}\right)=\left(e_{n-1}^{-1} \cdots e_{0}^{-1}\right)\left(\xi^{-}, \xi^{+}\right) .
$$

Since $\xi^{-}, \xi^{+} \notin \operatorname{Fix}\left(\Gamma_{0}\right)$,

$$
\gamma_{n}^{-1} e_{\sigma_{n-1}}^{-1} \cdots e_{\sigma_{0}}^{-1} \gamma_{0}=e_{n-1}^{-1} \cdots e_{0}^{-1} .
$$

According to the explicit formula for $\varphi$ (see [2]) there exists an absolute constant $N$ (that depends only on the group $\Gamma_{0}$ ) such that $\left|\gamma_{n}\right| \leq N$ for all $n$, where $|\cdot|$ denotes the word length with respect to the generating set $\left\{e_{a}\right\}$. Then

$$
\begin{aligned}
& d_{E_{G}}\left(\eta_{n}^{+}\left(\xi^{-}, \xi^{+}\right), \tau_{n}\left(\gamma_{0} \xi^{+}, \gamma_{0}\right)\right) \\
& =d_{E_{G}}\left(\gamma_{n}^{-1} e_{\sigma_{n-1}}^{-1} \cdots e_{\sigma_{0}}^{-1} \gamma_{0} \Gamma, e_{\sigma_{n-1}}^{-1} \cdots e_{\sigma_{0}}^{-1} \gamma_{0} \Gamma\right) \leq N .
\end{aligned}
$$

In particular,

$$
\lim _{n \rightarrow \infty} \eta_{n}^{+}\left(\xi^{-}, \xi^{+}\right)=\eta \Longleftrightarrow \lim _{n \rightarrow \infty} \tau_{n}\left(\gamma_{0} \xi^{+}, \gamma_{0}\right)=\eta .
$$

Observe that the right-hand term does not depend on $\xi^{-}$once $\gamma_{0}$ and $\xi^{+}$are given. Hence,

$$
A_{\gamma_{0}}=\left\{\xi^{+} \in \mathcal{D}: \lim _{n \rightarrow \infty} \tau_{n}\left(\gamma_{0} \xi^{+}, \gamma_{0}\right) \neq \eta\right\} .
$$

Since $\mathcal{D}$ is a $\Gamma_{0}$-invariant set,

$$
\gamma_{0} A_{\gamma_{0}}=\left\{\xi \in \mathcal{D}: \lim _{n \rightarrow \infty} \tau_{n}\left(\xi, \gamma_{0}\right) \neq \eta\right\}
$$


Let $\mu_{Y}$ be the measure on $Y=\partial \mathbb{D} \times G$ from Theorem 1.1 that satisfies equation (8) and $\mu(\cdot)=\mu_{Y}(\cdot \times\{\Gamma\})$. Then $\mu\left(A_{\gamma_{0}}\right)=\mu_{Y}\left(A_{\gamma_{0}} \times\{\Gamma\}\right)$. By equation (8),

$$
\mu_{Y}\left(A_{\gamma_{0}} \times\{\Gamma\}\right)=0 \Longleftrightarrow \mu_{Y}\left(\gamma_{0} A_{\gamma_{0}} \times\left\{\gamma_{0} \Gamma\right\}\right)=0 .
$$

Let $\mu_{X}=\mu_{Y} \circ \tilde{\pi}$, see Lemma 3.5. Then

$$
\mu_{Y}\left(\gamma_{0} A_{\gamma_{0}} \times\left\{\gamma_{0} \Gamma\right\}\right)=0 \Longleftrightarrow \mu_{X}\left(\pi_{\Sigma}^{-1}\left(\gamma_{0} A_{\gamma_{0}}\right) \times\left\{\gamma_{0} \Gamma\right\}\right)=0 .
$$

By equation (12),

$$
\pi_{\Sigma}^{-1}\left(\gamma_{0} A_{\gamma_{0}}\right)=\left\{\sigma^{+} \in \pi_{\Sigma}^{-1}(\mathcal{D}): \lim _{n \rightarrow \infty} T^{n}\left(\sigma, \gamma_{0}\right) \neq \pi_{S_{X}}^{-1}(\eta)\right\} .
$$

Then by Theorem 2.9 there exists $\eta \in \partial G$ such that $\pi_{\Sigma}^{-1}\left(\gamma_{0} A_{\gamma_{0}}\right) \times\left\{\gamma_{0} \Gamma\right\}$ is a $\mu_{X}$-null set.

Similarly, given $\eta \in \partial G$, let $\mu_{X}$ such that $\pi_{\Sigma}^{-1}\left(\gamma_{0} A_{\gamma_{0}}\right) \times\left\{\gamma_{0} \Gamma\right\}$ is a $\mu_{X}$-null set. Such a measure exists by Theorem 2.9 .

As for the other part of the proof, similar arguments show that, given $\mu \in$ $\operatorname{ext}(\operatorname{Conf}(\Gamma, \delta))$ with $\mu(\cdot)=\mu_{X}\left(\pi_{\Sigma}^{-1}(\cdot) \times\{\Gamma\}\right)$, we have that $\mu\left(A_{\gamma_{0}}\right)=0$.

Acknowledgments. I would like to thank my adviser Prof. Omri Sarig for professional and moral support. I would also like to express my gratitude to Uri Shapira for the warm hospitality during my stay at the Technion. Lastly, I thank Manuel Stadlbauer and Jon Aaronson for useful discussions and the referee for helpful comments. The author acknowledges the support of ISF grants $871 / 17$ and 1149/18. This work has received funding from the European Research Council (ERC) under the European Union's Horizon 2020 research and innovation programme (Grant Agreement No. 754475).

\section{A. Appendix. The Martin boundary of a Markov shift over a hyperbolic graph}

A.1. Discretized Green function and related inequalities. To prove Theorem 2.9 (the generalized Ancona theorem), we introduce approximated versions Green's function and the Martin kernel to the discrete set of states $S$ rather than the non-discrete set of infinite paths $X$. For these discretized functions, we present several combinatorial inequalities, inspired by their probabilistic analogues.

Recall that for every $a \in S$ we fixed $x_{a} \in T[a]$ arbitrarily.

Definition A.1. For $a, b \in S$ and $\lambda>0$, let

$$
G(a, b \mid \lambda):=G\left(1_{[a]}, b x_{b} \mid \lambda\right)=\sum_{n=0}^{\infty} \sum_{\substack{\left(a_{0}, \ldots, a_{n}\right) \in \mathcal{W}^{n+1} \\ a_{0}=a, a_{n}=b}} \lambda^{-n} e^{\phi_{n}\left(a_{0}, \ldots, a_{n} x_{b}\right)}
$$

and let

$$
F(a, b \mid \lambda):=\sum_{n=0}^{\infty} \sum_{\begin{array}{c}
\left(a_{0}, \ldots, a_{n}\right) \in \mathcal{W}^{n+1} \\
a_{0}=a, a_{n}=b \\
\text { for all } i<n, a_{i} \neq b
\end{array}} \lambda^{-n} e^{\phi_{n}\left(a_{0}, \ldots, a_{n} x_{b}\right)} .
$$

We let $\phi_{0} \equiv 0$. In particular, $F(a, a \mid \lambda)=1$. 
For a subset $A \subseteq S$, we let

$$
L^{A}(a, b \mid \lambda):=\sum_{n=0}^{\infty} \sum_{\begin{array}{c}
\left(a_{0}, \ldots, a_{n}\right) \in \mathcal{W}^{n+1} \\
a_{0}=a, a_{n}=b \\
a_{0} \in A, \text { for all } i>0: a_{i} \notin A
\end{array}} \lambda^{-n} e^{\phi_{n}\left(a_{0}, \ldots, a_{n} x_{b}\right)}
$$

and let

$$
F^{A}(a, b \mid \lambda):=\sum_{n=0}^{\infty} \sum_{\substack{\left(a_{0}, \ldots, a_{n}\right) \in \mathcal{W}^{n+1} \\ a_{0}=a, a_{n}=b \\ a_{n} \in A, \text { for all } i<n: a_{i} \notin A}} \lambda^{-n} e^{\phi_{n}\left(a_{0}, \ldots, a_{n} x_{b}\right)}
$$

Observe that if $a \notin A$ then $L^{A}(a, b \mid \lambda)=0$ and if $b \notin A$ then $F^{A}(a, b \mid \lambda)=0$. We write $L^{\{a\}}(a, b \mid \lambda)=L(a, b \mid \lambda)$. Let

$$
K(a, b \mid \lambda):=K\left(1_{[a]}, b x_{b} \mid \lambda\right)=\frac{G(a, b \mid \lambda)}{G(o, b \mid \lambda)}
$$

and given $f \in C_{c}(X)$, let $K(f, a \mid \lambda):=K\left(f, a x_{b} \mid \lambda\right)$. For $\lambda=1$, we simply write $G(a, b), F(a, b), L(a, b)$ and $K(a, b)$.

The following propositions present several useful inequalities involving the functions $F, G, L$ and $K$. Their proofs are elementary and are partly included here. Several of these inequalities have been adapted from probabilistic settings; see [54] for more details.

Proposition A.2. Assume that $(X, T)$ is locally compact and transitive, that $\phi$ has summable variations and that $P_{G}(\phi)<\infty$. Then there exists a constant $C>1$ such that, for every $\lambda>\rho(\phi)$, the following statements hold.

(1) For every $a, b \in S$,

$$
G(a, b \mid \lambda)=C^{ \pm 1} F(a, b \mid \lambda) G(b, b \mid \lambda) .
$$

(2) For every $a, b, c \in S$,

$$
F(a, c \mid \lambda) F(c, b \mid \lambda) \leq C F(a, b \mid \lambda) .
$$

(3) For every $a, b \in S$ and every set $A \subseteq S$ such that every path from a to $b$ must pass through A,

$$
G(a, b \mid \lambda)=C^{ \pm 1} \sum_{e \in A} G(a, e \mid t) L^{A}(e, b \mid \lambda) .
$$

(4) For every $a, b \in S$ and every set $A \subseteq S$,

$$
\sum_{e \in A} G(a, e \mid \lambda) L^{A}(e, b \mid \lambda)=C^{ \pm 1} \sum_{e \in A} F^{A}(a, e \mid \lambda) G(e, b \mid \lambda) .
$$

(5) For every $a, b \in S$ and $A \subseteq S$,

$$
\sum_{e \in A} F^{A}(a, e \mid \lambda) G(e, b \mid \lambda) \leq C G(a, b \mid \lambda) .
$$


(6) For every $a, b \in S$ and every $\lambda_{1}, \lambda_{2}$ with $\rho(\phi)<\lambda_{1} \leq \lambda_{2}$,

$$
\frac{G\left(a, b \mid \lambda_{1}\right)}{\lambda_{1}}-\frac{G\left(a, b \mid \lambda_{2}\right)}{\lambda_{2}}=C^{ \pm 1}\left(\frac{1}{\lambda_{1}}-\frac{1}{\lambda_{2}}\right) \sum_{c \in S} G\left(a, c \mid \lambda_{1}\right) G\left(c, b \mid \lambda_{2}\right) .
$$

(7) For every admissible $a_{1}, \ldots, a_{N} \in S$ and every $b_{1}, b_{2} \in S$ with $b_{i} \neq a_{j}$,

$$
K\left(1_{\left[a_{1}, \ldots, a_{N}\right]}, b_{i} \mid \lambda\right)=C^{ \pm 1} t^{-(N-1)} e^{\phi_{N-1}\left(a_{1}, \ldots, a_{N} x_{a_{N}}\right)} K\left(a_{N}, b_{i} \mid \lambda\right)
$$

and

$$
\frac{K\left(1_{\left[a_{1}, \ldots, a_{N}\right]}, b_{1} \mid \lambda\right)}{K\left(1_{\left[a_{1}, \ldots, a_{N}\right]}, b_{2} \mid \lambda\right)}=C^{ \pm 1} \frac{F\left(a_{N}, b_{1} \mid t\right) F\left(o, b_{2} \mid \lambda\right)}{F\left(a_{N}, b_{2} \mid t\right) F\left(o, b_{1} \mid \lambda\right)} .
$$

Proposition A.3. Assume that $(X, T)$ is locally compact and transitive, that $\phi$ has summable variations, that $\phi$ is uniformly irreducible with respect to a connected, undirected and locally finite graph $(S, E)$ and that $P_{G}(\phi)<0$. Then the following statements hold.

(1) For every $a, b \in S$ and every $\lambda \in(\rho(\phi), 1)$,

$$
L^{A}(a, b \mid \lambda) \geq \lambda^{-d_{E}(a, b)} L^{A}(a, b) .
$$

(2) (Harnack's inequality) There exists $C^{\prime}>1$ such that for every $\lambda \in(\rho(\phi), 1]$, for every $h \in\{G(\cdot, c \mid \lambda), F(\cdot, c \mid \lambda), G(c, \cdot \mid \lambda), F(c, \cdot \mid \lambda)\}_{c \in S}$ and every $a, b \in S$,

$$
h(a) \leq\left(C^{\prime}\right)^{d_{E}(a, b)} h(b) .
$$

Proof. The proof of (1) is elementary and follows directly from the definition of the function $L^{A}$. We prove (2).

Let $K>0$ such that for every $(a, b) \in E$ there exists $k \leq K$ with $L_{\phi}^{k}\left(1_{[a]}\right)\left(b x_{b}\right)>$ 0 and let $\epsilon=\exp \left(-K \min _{x \in X}|\phi(x)|\right)$. Let $N=d_{E}(a, b)+1$ and let $a_{1}, \ldots, a_{N}$ be a shortest path in $E$ from $a_{1}=b$ to $a_{N}=a$. Let $k_{1}, \ldots, k_{N-1} \geq 1$ with $k_{i} \leq K$ and $L_{\phi}^{k_{i}}\left(1_{\left[a_{i}\right]}\right)\left(a_{i+1} x_{a_{i+1}}\right)>0$. Let $k=\sum_{i=1}^{N-1} k_{i}$. Notice that $d_{E}(a, b) \leq k \leq d_{E}(a, b) K$. Then

$$
\begin{aligned}
G(b, c \mid \lambda) & \geq \sum_{n=k}^{\infty} \lambda^{-n} L_{\phi}^{n}\left(1_{[b]}\right)\left(c x_{c}\right) \\
& \geq \lambda^{-k} \sum_{n=0}^{\infty} \lambda^{-n} L_{\phi}^{n+k}\left(1_{[b]} \cdot 1_{T^{-d_{E}(a, b)}[a]}\right)\left(c x_{c}\right) \\
& \geq \lambda^{-k} C_{\phi}^{-k} \sum_{n=0}^{\infty} L_{\phi}^{k_{1}}\left(1_{[b]}\right)\left(a_{2} x_{a_{2}}\right) \cdots L_{\phi}^{k_{N-1}}\left(1_{\left[a_{N-1}\right]}\right)\left(a x_{a}\right) \lambda^{-n} L_{\phi}^{n}\left(1_{[a]}\right)\left(c x_{c}\right) \\
& \geq \lambda^{-k} C_{\phi}^{-k} \epsilon_{0}^{d_{E}(a, b)} G(a, c \mid \lambda) \\
& \geq\left(\max \left\{C_{\phi}, C_{\phi}^{K}\right\} \epsilon^{-1}\right)^{-d_{E}(a, b)} G(a, c \mid \lambda) .
\end{aligned}
$$

So, with $B=\max \left\{C_{\phi}, C_{\phi}^{K}\right\} \epsilon_{0}^{-1}$,

$$
G(a, c \mid \lambda) \leq B^{d_{E}(a, b)} G(b, c \mid \lambda) .
$$


Similar arguments lead to $G(c, b \mid \lambda) \leq B^{d_{E}(a, b)} G(c, a \mid \lambda)$.

Let $C>1$ be the constant from Proposition A.2. Then

$$
\begin{aligned}
F(a, c \mid \lambda) & \geq C^{-1} \frac{G(a, c \mid \lambda)}{G(c, c \mid \lambda)} \\
& \geq C^{-1} B^{-d_{E}(a, b)} \frac{G(b, c \mid \lambda)}{G(c, c \mid \lambda)} \\
& \geq C^{-2} B^{-d_{E}(a, b)} F(b, c \mid \lambda) .
\end{aligned}
$$

Moreover, since $G(a, a \mid \lambda) \geq B^{-d_{E}(a, b)} G(a, b \mid \lambda)$ and $G(b, b \mid \lambda) \leq B^{d_{E}(a, b)} G(a, b \mid \lambda)$, we have that

$$
\frac{G(a, a \mid \lambda)}{G(b, b \mid \lambda)} \geq B^{-2 d_{E}(a, b)}
$$

We conclude that

$$
\begin{aligned}
F(c, b \mid \lambda) & \geq C^{-1} \frac{G(c, b \mid \lambda)}{G(b, b \mid \lambda)} \\
& \geq C^{-1} B^{-d_{E}(a, b)} \frac{G(c, a \mid \lambda)}{G(b, b \mid \lambda)} \\
& \geq C^{-2} B^{-d_{E}(a, b)} F(c, a \mid \lambda) \frac{G(a, a \mid \lambda)}{G(b, b \mid \lambda)} \\
& \geq C^{-2} B^{-3 d_{E}(a, b)} F(c, a \mid \lambda) .
\end{aligned}
$$

A.2 Proof of Theorem 2.9. We follow here the arguments of the proof of the original theorem as presented in [54].

Observe that if $\lambda>\rho(\phi)$ then $P_{G}(\phi-\log \lambda)<0$ and that for all $f \in C_{c}(X)$ and $x \in X$,

$$
\lambda^{-n}\left(L_{\phi}^{n} f\right)(x)=L_{\phi-\log \lambda}^{n}(f)(x) .
$$

Thus we can assume without loss of generality that $P_{G}(\phi)<0$ and prove the theorem for $\lambda=1$.

In what follows, assume that $(S, E)$ is a $\delta$-hyperbolic graph and that $P_{G}(\phi)<0$. For $a, b \in S$, let

$$
U_{a, b}=\left\{c \in S:|b \wedge c|_{a} \geq d_{E}(a, b)-7 \delta\right\}
$$

and let $V_{b, a}=S \backslash U_{a, b}$. For $a \in S$ and $r \geq 0$, we denote by $B(a, r)=\left\{b \in S: d_{E}(a, b) \leq\right.$ $r$ \} the closed ball of radius $r$ around $a$. Let $C, C^{\prime}>1$ be the constants from Propositions A. 2 and A. 3 respectively and let $C_{0}=\max \left\{C, C^{\prime}\right\}$.

PROPOSITION A.4. Under the assumptions of Theorem 2.9, for every $\lambda \in(\rho(\phi), 1)$ there exists a constant $C_{1}(\lambda)>1$ such that for every $a, b \in S$ and for every $v$ on some geodesic segment in the graph $(S, E)$ from a to $b$,

$$
G(a, w) \leq C_{1}(t) F(a, v) G(v, w \mid \lambda) \quad \text { for all } w \in U_{a, v} \cup V_{v, b} .
$$

Proof. We use only properties that do not depend on the base point and so we can assume without loss of generality that $a=o$. Let $\ell=21 \delta$, let $m$ be the integer part of $d(o, v) / \ell$ 
( $m$ may be zero) and consider the points $v_{0}, \ldots, v_{m}$ which lie on a geodesic segment between $o$ and $v$ with $d_{E}\left(v_{k}, v\right)=(m-k) \ell$. Let $W_{k}=U_{o, v_{k}} \cup V_{v_{k}, b}$ and let $d W_{k}=\{w \in$ $\left.W_{k}: d_{E}\left(w, S \backslash W_{k}\right)=1\right\}$.

Lemma A.5. Assume that $(S, E)$ is a $\delta$-hyperbolic graph. Then, for all $k \geq 1$, the following statements hold.

(1) $v_{k} \in W_{k} \subseteq W_{k-1}$.

(2) If $w \in W_{k}$ with $d_{E}\left(w, v_{k}\right) \geq 2 r+\ell+1$ then $B(w, r) \subseteq W_{k-1}$.

Proof. See [54, Lemma 27.7].

Choose an integer $r \geq \ell$ with $\lambda^{r} C_{0}^{2 l+4} \leq 1$. We show by induction on $k$ that, with $C_{1}=C_{0}^{4 r+2 \ell+1}$,

$$
G(o, w) \leq C_{1} F\left(o, v_{k}\right) G\left(v_{k}, w \mid \lambda\right) \quad \text { for all } w \in W_{k} .
$$

The proposition follows with $k=m$.

Let $k=0$. Then $d_{E}\left(o, v_{0}\right) \leq \ell$ and by Proposition A.3.2, for every $w \in S$,

$$
G(o, w) \leq C_{0}^{\ell} G\left(v_{0}, w\right) \leq C_{0}^{\ell} G\left(v_{0}, w \mid \lambda\right) .
$$

Similarly, $G\left(v_{0}, v_{0}\right) \leq C_{0}^{\ell} G\left(o, v_{0}\right)$. By Proposition A.2.1,

$$
F\left(o, v_{0}\right) \geq C_{0}^{-1} \frac{G\left(o, v_{0}\right)}{G\left(v_{0}, v_{0}\right)} \geq C_{0}^{-\ell-1}
$$

and thus, for every $w \in S, G(o, w) \leq C_{0}^{2 \ell+1} F\left(o, v_{0}\right) G\left(v_{0}, w \mid \lambda\right)$.

Next, suppose by induction that equation (A.1) holds for $k-1$. Since $d_{E}\left(v_{k-1}, v_{k}\right) \leq \ell$, by Proposition A.3.2, for all $w \in S$,

$$
G\left(v_{k-1}, w \mid \lambda\right) \leq C_{0}^{\ell} G\left(v_{k}, w \mid \lambda\right) .
$$

Similarly to equation (A.2),

$$
C_{0}^{\ell+1} F\left(v_{k-1}, v_{k}\right) \geq 1
$$

Hence, for all $w \in W_{k-1}$,

$$
\begin{aligned}
G(o, w) & \leq C_{1} F\left(o, v_{k-1}\right) G\left(v_{k-1}, w \mid \lambda\right) & & (\because \text { induction hypothesis }) \\
& \leq C_{1} C_{0}^{2 \ell+1} F\left(o, v_{k-1}\right) F\left(v_{k-1}, v_{k}\right) G\left(v_{k}, w \mid \lambda\right) & & (\because \text { equations (A.3), (A.4) }) \\
& \leq C_{1} C_{0}^{2 \ell+2} F\left(o, v_{k}\right) G\left(v_{k}, w \mid \lambda\right) & & (\because \text { Proposition A.2.2). }
\end{aligned}
$$

Now, let $w \in W_{k}$ and assume first that $d_{E}\left(w, v_{k}\right) \geq 2 r+\ell+1$. Set $A=\{e \in S$ : $d_{E}(e, w)=r$. By Lemma A.5, $A \subseteq W_{k-1}$ and hence equation (A.5) holds for all $e \in A$. We claim that any path from $o$ to $w$ must pass through $A$. If $k=1$ then, by construction, $d_{E}\left(o, v_{1}\right) \geq \ell$ and, by assumption, $d_{E}\left(w, v_{1}\right) \geq 2 r+\ell+1$ so $d_{E}(o, w)>r$. Thus any path from $o$ to $w$ must enter $A$. Observe that $o \notin W_{1}$ and, by Lemma A.5, $o \notin W_{k}$ for all $k$. Hence, if $k \geq 2$ then $o \notin B(w, r)$ and again any path from $o$ from $w$ must enter $A$. 
We deduce that

$$
\begin{aligned}
G(o, w) & \leq C_{0} \sum_{e \in A} G(o, e) L^{A}(e, w) \\
& \leq C_{1} C_{0}^{2 \ell+3} \sum_{e \in A} F\left(o, v_{k}\right) G\left(v_{k}, e \mid \lambda\right) L^{A}(e, w)
\end{aligned}
$$

Since $d_{E}\left(w, v_{k}\right)>r$, any path from $w$ to $v_{k}$ must pass through $A$. Therefore, by Proposition A.2.3,

$$
C_{1} C_{0}^{2 \ell+3} \lambda^{r} \sum_{e \in A} F\left(o, v_{k}\right) G\left(v_{k}, e \mid \lambda\right) L^{A}(e, w \mid \lambda) \leq C_{1} C_{0}^{2 \ell+4} t^{r} F\left(o, v_{k}\right) G\left(v_{k}, w \mid \lambda\right) .
$$

So, for all $w \in W_{k}$ with $d_{E}\left(w, v_{k}\right) \geq 2 r+\ell+1$,

$$
G(o, w) \leq C_{1} C_{0}^{2 \ell+4} \lambda^{r} F\left(o, v_{k}\right) G\left(v_{k}, w \mid \lambda\right) .
$$

By the choice of $r$, equation (A.1) follows.

Lastly, if $w \in W_{k}$ with $d_{E}\left(w, v_{k}\right) \leq 2 r+\ell$, then

$$
\begin{aligned}
G(o, w) & \leq C_{0}^{2 r+\ell} G\left(o, v_{k}\right) & & (\because \text { Proposition A.3.2 }) \\
& \leq C_{0}^{2 r+\ell+1} F\left(o, v_{k}\right) G\left(v_{k}, v_{k}\right) & & (\because \text { Proposition A.2.1 }) \\
& \leq C_{0}^{2 r+\ell+1} F\left(o, v_{k}\right) G\left(v_{k}, v_{k} \mid \lambda\right) & & (\because \lambda<1) \\
& \leq C_{0}^{4 r+2 \ell+1} F\left(o, v_{k}\right) G\left(v_{k}, w \mid \lambda\right) & & (\because \text { Proposition A.3.2 }) .
\end{aligned}
$$

COROLlARY A.6. Under the assumptions of Theorem 2.9, for every $\lambda \in(\rho(\phi), 1)$ there is a constant $C_{2}(\lambda)>1$ such that for every $a, b \in S$ and for every $v$ on some geodesic segment in the graph $(S, E)$ from a to $b$,

$$
G(w, b) \leq C_{2}(\lambda) G(w, v \mid \lambda) L(v, b) \quad \text { for all } w \in V_{v, a} \cup U_{b, v} .
$$

Proof. Denote by $X^{ \pm}$is the two-sided shift, by $X^{-}$the negative one-sided shift and by $X^{+}=X$ the positive one-sided shift. It is known that there exist a potential function $\phi^{-}$: $X^{-} \rightarrow \mathbb{R}$ with summable variations and a bounded uniformly continuous function $\psi$ : $X^{ \pm} \rightarrow \mathbb{R}$ such that

$$
\phi^{+}-\phi^{-}=\psi-\psi \circ T
$$

see $[49, \S 5]$. We add the + or - notation over Green's functions to distinguish between the two spaces (e.g. $G^{+}$or $G^{-}$).

LEMma A.7. Assume that $(X, T)$ is locally compact and transitive, that $\phi$ has summable variations and that $P_{G}(\phi)<\infty$. Then there exists $C^{\prime \prime}>1$ such that for every $\lambda>\rho(\phi)$ and every $a, b, c \in S$,

$$
G^{-}(a, b \mid \lambda)=\left(C^{\prime \prime}\right)^{ \pm 1} G^{+}(b, a \mid \lambda)
$$

and

$$
F^{-}(a, b) G^{-}(b, c \mid \lambda)=\left(C^{\prime \prime}\right)^{ \pm 1} G^{+}(c, b \mid \lambda) L^{+}(b, a \mid \lambda)
$$


Proof. Let $a, b \in S$, let $a_{1}, \ldots, a_{n-1}$ be an admissible path from $a$ to $b$, let $x_{a}^{-} \in[a] \subseteq$ $X^{-}$and let $x_{b}^{+} \in[b] \subseteq X^{+}$. Since $\phi^{-}, \phi^{+}$and $\psi$ are all bounded,

$$
\begin{aligned}
& \left|\phi_{n}^{+}\left(a, a_{1}, \ldots, a_{n-1} x_{b}^{+}\right)-\phi_{n}^{-}\left(x_{a}^{-} a_{1}, \ldots, a_{n-1}, b\right)\right| \\
& \leq\left|\phi^{+}\left(a, a_{1}, \ldots, a_{n-1} x_{b}^{+}\right)\right|+\left|\phi^{-}\left(x_{a}^{-} a_{1}, \ldots, a_{n-1}, b\right)\right| \\
& \quad+\left|\sum_{i=1}^{n-1}\left(\phi^{+}\left(a_{i}, \ldots, a_{n-1} x_{b}^{+}\right)-\phi^{-}\left(x_{a}^{-} a_{1}, \ldots, a_{i}\right)\right)\right| \\
& \quad \leq \sup \left|\phi^{+}\right|+\sup \left|\phi^{-}\right|+2 \sup |\psi| .
\end{aligned}
$$

Now any path from $a$ to $b$ in $X^{+}$is a path from $b$ to $a$ in $X^{-}$and there thus is a natural matching of the terms in the sums $G^{+}, G^{-}$and $F^{+}, F^{-}$with the property that matching terms are within multiplicative error $e^{ \pm\left(\sup \left|\phi^{+}\right|+\sup \left|\phi^{-}\right|+2 \sup |\psi|\right)}$ of each other.

The corollary follows from Proposition A.4 and Lemma A.7.

THEOREM A.8. (Ancona's inequality) Under the assumptions of Theorem 2.9, for every $r \geq 0$ there exists $C_{3}(r) \geq 1$ such that

$$
\left(C_{3}(r)\right)^{-1} F(a, v) F(v, b) \leq F(a, b) \leq C_{3}(r) F(a, v) F(v, b)
$$

whenever $a, b \in S$ and $v$ is at distance at most $r$ from some geodesic segment from $a$ to $b$ in the graph $(S, E)$.

Proof. The lower bound follows from Proposition A.2.2, so we focus on the upper bound. We first consider the case $r=0$, when $v$ lies on a geodesic segment from $a$ to $b$.

Fix $\lambda \in((\rho(\phi)+1) / 2,1)$. If $d_{E}(a, v) \leq 7 \delta$ then by Proposition A.3.2, $F(a, b) \leq$ $C_{0}^{7 \delta} F(v, b)$ and $1 \leq F(v, v) \leq C_{0}^{7 \delta} F(a, v)$. In particular, $F(a, b) \leq C_{0}^{14 \delta} F(a, v) F(v, b)$.

Suppose that $d_{E}(a, v)>7 \delta$. Since $|a \wedge v|_{a}=0, a \notin U_{a, v}$. Moreover, since $v$ lies on a geodesic segment from $a$ to $b,|v \wedge b|_{a}=d_{E}(a, v)$ and thus $b \in U_{a, v}$. In particular, any path from $a$ to $b$ must pass through

$$
A:=\left\{c \in U_{a, v}: \exists w \in S \backslash U_{a, v}, d_{E}(c, w)=1\right\} .
$$

By Propositions A.2.3 and A.4,

$$
G(a, b) \leq C_{0} \sum_{w \in A} G(a, w) L^{A}(w, b) \leq C_{0} C_{1} F(a, v) \sum_{w \in A} G(v, w \mid \lambda) L^{A}(w, b)
$$

where $C_{1}=C_{1}(1)$ is the constant from Proposition A.4. Every point $w \in A$ is at distance 1 from some point $w^{\prime}$ in $V_{v, a}=S \backslash U_{a, v}$. By Corollary A.6,

$$
G\left(w^{\prime}, b\right) \leq C_{2} G\left(w^{\prime}, v \mid \lambda\right) L(v, b)
$$

where $C_{2}=C_{2}(1)$ is the constant from Corollary A.6. We apply Proposition A.3.2 to $G\left(w^{\prime}, b\right)$ and $G\left(w^{\prime}, v \mid \lambda\right)$ to obtain that

$$
G(w, b) \leq C_{2} C_{0}^{2} G(w, v \mid \lambda) L(v, b) .
$$


Then, by Proposition A.2, for every $e \in S$,

$$
\begin{aligned}
\sum_{w \in A} G(e, w) L^{A}(w, b) \leq & C_{0} \sum_{w \in A} F^{A}(e, w) G(w, b) & & (\because \text { Proposition A.2.4) } \\
\leq & C_{0} \sum_{w \in A} F^{A}(e, w \mid \lambda) G(w, b) & & (\because \lambda<1) \\
\leq & C_{2} C_{0}^{3} \sum_{w \in A} F^{A}(e, w \mid \lambda) & & \\
& \times G(w, v \mid \lambda) L(v, b) & & (\because \text { equation (A.7) }) \\
\leq & C_{2} C_{0}^{4} G(e, v \mid \lambda) L(v, b) & & (\because \text { Proposition A.2.5). }
\end{aligned}
$$

Let $v(e)=\lambda \delta_{v}(e)+(1-\lambda) G(v, e \mid \lambda)$. By Proposition A.2.6, with $\lambda_{1}=1$ and $\lambda_{2}=\lambda$.

$$
\begin{aligned}
G(v, w \mid \lambda) & \leq \lambda G(v, w)+C_{0}(1-\lambda) \sum_{e \in S} G(v, e \mid \lambda) G(e, w) \\
& \leq C_{0}\left(\lambda G(v, w)+(1-\lambda) \sum_{e \in S} G(v, e \mid \lambda) G(e, w)\right) \\
& =C_{0} \sum_{e \in S} v(e) G(e, w) .
\end{aligned}
$$

In summary,

$$
\begin{aligned}
G(a, b) & \leq C_{0} C_{1} F(a, v) \sum_{w \in A} G(v, w \mid \lambda) L^{A}(w, b) \\
& \leq C_{0}^{2} C_{1} F(a, v) \sum_{w \in A} \sum_{e \in S} v(e) G(e, w) L^{A}(w, b) \\
& =C_{0}^{2} C_{1} F(a, v) \sum_{e \in S} v(e)\left(\sum_{w \in A} G(e, w) L^{A}(w, b)\right) \\
& \leq C_{0}^{6} C_{1} C_{2} F(a, v)\left(\sum_{e \in S} v(e) G(e, v \mid \lambda)\right) L(v, b)
\end{aligned}
$$

Choose $\lambda_{2}(\lambda) \in(\rho(\phi), 2 \lambda-1)$ with $\lim _{\lambda \rightarrow 1^{-}} \lambda_{2}(\lambda)=1$. Then, $1-\lambda \leq \lambda-\lambda_{2}$ and

$$
\begin{aligned}
\sum_{e \in S} v(e) G(e, v \mid \lambda) & =\lambda G(v, v \mid \lambda)+(1-\lambda) \sum_{e \in S} G(v, e \mid \lambda) G(e, v \mid \lambda) \\
& \leq \lambda G(v, v \mid \lambda)+\left(\lambda-\lambda_{2}\right) \sum_{e \in S} G\left(v, e \mid \lambda_{2}\right) G(e, v \mid \lambda) \\
& \leq \lambda G(v, v \mid \lambda)+C_{0} \frac{\lambda-\lambda_{2}}{1 / \lambda_{2}-1 / \lambda}\left(\frac{G\left(v, v \mid \lambda_{2}\right)}{\lambda_{2}}-\frac{G(v, v \mid \lambda)}{\lambda}\right) \\
& \leq \lambda G(v, v \mid \lambda)+C_{0} t G\left(v, v \mid \lambda_{2}\right) \\
& \leq C_{0} t\left(G(v, v \mid \lambda)+G\left(v, v \mid \lambda_{2}\right)\right) .
\end{aligned}
$$

This leads to

$$
G(a, b) \leq C_{0}^{7} C_{1} C_{2} t F(a, v)\left(G(v, v \mid \lambda)+G\left(v, v \mid \lambda_{2}\right)\right) L(v, b) .
$$


Since $\rho(\phi)<1, G(v, v \mid \lambda)$ is analytic as a function of $\lambda$ on a neighborhood of $\lambda=1$ and

$$
\lim _{\lambda \nearrow 1} G(v, v \mid \lambda)=\lim _{\lambda \nearrow 1} G\left(v, v \mid \lambda_{2}(\lambda)\right)=G(v, v) .
$$

Therefore

$$
G(a, b) \leq 2 C_{0}^{7} C_{1} C_{2} F(a, v) G(v, v) L(v, b) .
$$

By Proposition A.2.3,

$$
G(a, b) \leq 2 C_{0}^{7} C_{1} C_{2} F(a, v) G(v, b) .
$$

We divide both sides by $G(b, b)$ and apply Proposition A.2.1 to obtain that

$$
F(a, b) \leq 2 C_{0}^{9} C_{1} C_{2} F(a, v) F(v, b) .
$$

This proves Ancona's inequality in the case wherev lies on a geodesic segment from $a$ to $b$.

Now assume that $v$ is at distance $r \geq 0$ from some geodesic segment from $a$ to $b$. Then we can find $v^{\prime} \in S$ on this geodesic segment from $a$ to $b$ with $d\left(v, v^{\prime}\right)=r$. By the first part of the proof,

$$
F(a, b) \leq 2 C_{0}^{9} C_{1} C_{2} F\left(a, v^{\prime}\right) F\left(v^{\prime}, b\right) .
$$

Applying Proposition A.3.2 twice leads to

$$
F(a, b) \leq 2 C_{0}^{9+2 r} C_{1} C_{2} F(a, v) F(v, b) .
$$

Proof of Theorem 2.9. Let $\xi \in \partial(S, E)$. We first show that there exists $\epsilon_{1} \in(0,1)$ such that for every $f \in C_{c}^{+}(X)$ and for every two sequences $b_{n}, b_{n}^{\prime} \in S$ which converge to $\xi$,

$$
\liminf _{n \rightarrow \infty} K\left(f, b_{n}\right) \geq \epsilon_{1} \limsup _{n \rightarrow \infty} K\left(f, b_{n}^{\prime}\right) .
$$

LEMmA A.9. Let $(S, E)$ be a $\delta$-hyperbolic graph. Then, for every $n$, there exists $v_{n} \in S$ which is at distance at most $2 \delta$ from some geodesic segments from a to $b_{n}$, from o to $b_{n}$, from a to $b_{n}^{\prime}$, and from o to $b_{n}^{\prime}$.

Proof. See [54], proof of Theorem 27.1.

For every $n>0$, let $v_{n} \in S$ as in the lemma and let $a_{1}, \ldots, a_{N} \in S$ with $\left[a_{1}, \ldots, a_{N}\right] \neq \varnothing$. By Proposition A.2.7 and Theorem A.8, for all $n$ large enough,

$$
\begin{aligned}
\frac{K\left(1_{\left[a_{1}, \ldots, a_{N}\right]}, b_{n}\right)}{K\left(1_{\left[a_{1}, \ldots, a_{N}\right]}, b_{n}^{\prime}\right)} & \geq C_{0}^{-1} \frac{F\left(a, b_{n}\right) F\left(o, b_{n}^{\prime}\right)}{F\left(a, b_{n}^{\prime}\right) F\left(o, b_{n}\right)} \\
& \geq C_{0}^{-1}\left(C_{3}(2 \delta)\right)^{-4} \frac{F\left(a, v_{n}\right) F\left(v_{n}, b_{n}\right) F\left(o, v_{n}\right) F\left(v_{n}, b_{n}^{\prime}\right)}{F\left(a, v_{n}\right) F\left(v_{n}, b_{n}^{\prime}\right) F\left(o, v_{n}\right) F\left(v_{n}, b_{n}\right)} \\
& =C_{0}^{-1}\left(C_{3}(2 \delta)\right)^{-4} .
\end{aligned}
$$

Here $C_{3}(2 \delta)$ is the constant in Ancona's inequality for $r=2 \delta$. In particular,

$$
\liminf _{n \rightarrow \infty} K\left(1_{\left[a_{1}, \ldots, a_{N}\right]}, b_{n}\right) \geq C_{0}^{-1}\left(C_{3}(2 \delta)\right)^{-4} \limsup _{n \rightarrow \infty} K\left(1_{\left[a_{1}, \ldots, a_{N}\right]}, b_{n}^{\prime}\right) .
$$


Since the collection of cylinder sets linearly spans a dense subset of $C_{c}(X)$ with respect to the sup norm, equation (A.10) extends to all $f \in C_{c}^{+}(X)$.

Let $\epsilon_{1}=C_{0}^{-1}\left(C_{3}(2 \delta)\right)^{-4} \in(0,1)$ and let

$$
\mathcal{A}_{\xi}=\left\{\omega: \exists b_{n} \in S \text { such that } b_{n} \rightarrow \xi \text { and } \lim _{n \rightarrow \infty} K\left(f, b_{n}\right)=K(f, \omega), \forall f \in C_{c}(X)\right\} \text {, }
$$

that is, the set of all possible limit points in $\mathcal{M}$ of sequences $b_{n} \in S$ with $b_{n} \rightarrow \xi$ in $(S, E)$. We show that $\mathcal{A}_{\xi}$ consists of a single point alone. Notice that for all $\omega_{1}, \omega_{2} \in \mathcal{A}_{\xi}$,

$$
K\left(f, \omega_{1}\right) \geq \epsilon_{1} K\left(f, \omega_{2}\right) \text { for all } f \in C_{c}^{+}(X) .
$$

Therefore, it suffices to show that $\mathcal{A}_{\xi} \cap \mathcal{M}_{m} \neq \varnothing$.

Let $\omega \in \mathcal{A}_{\xi}$,

$$
\mathcal{C}=\left\{\mu \text { Radon }: \mu \geq 0 \text { and } L_{\phi}^{*} \mu=\mu\right\}
$$

and let

$$
\mathcal{B}_{\omega}=\left\{\mu \in \mathcal{C}: \sup _{f \in C_{c}^{+}(X)} \frac{\mu(f)}{\mu_{\omega}(f)}=1\right\} .
$$

Recall that $\mu_{\omega}(f)=K(f, \omega), f \in C_{c}(X)$. If $\mu_{\omega}=\mu_{1}+\mu_{2}$ with $\mu_{i} \in \mathcal{C}$ and the measures $\mu_{1}, \mu_{2}$ are mutually singular and non-zero then $\sup _{f \in C_{c}^{+}(X)}\left\{\mu_{i} / \mu_{\omega}\right\}=1$. Thus, it suffices to show that $\mathcal{B}_{\omega}=\left\{\mu_{\omega}\right\}$.

Let $\left(b_{0}, b_{1}, \ldots\right)$ be a geodesic sequence converging to $\xi$ in $(S, E)$ with $b_{0}=$ $o$ and $\lim _{n \rightarrow \infty} K\left(f, b_{n}\right)=K(f, \omega)$ for every $f \in C_{c}(X)$. By Proposition A.2.1 and Theorem A.8,

$$
K\left(b_{k}, b_{n}\right) \geq C_{0}^{-2} \frac{F\left(b_{k}, b_{n}\right)}{F\left(o, b_{n}\right)} \geq C_{0}^{-2} C_{3}^{-1} \frac{1}{F\left(o, b_{k}\right)},
$$

where $C_{3}=C_{3}(0)$. Therefore, for every $a \in S$,

$$
K\left(a, b_{k}\right) \leq C_{0}^{2} \frac{F\left(a, b_{k}\right)}{F\left(o, b_{k}\right)} \leq C_{3} C_{0}^{4} F\left(a, b_{k}\right) K\left(b_{k}, b_{n}\right) \underset{n \rightarrow \infty}{\longrightarrow} C_{3} C_{0}^{4} F\left(a, b_{k}\right) K\left(b_{k}, \omega\right) .
$$

Let $a_{1}, \ldots, a_{N} \in S$ be admissible. By Proposition A.2.7, for all $k$ large enough,

$$
\begin{aligned}
K\left(1_{\left[a_{1}, \ldots, a_{N}\right]}, b_{k}\right) & \leq C_{0} e^{\phi_{N-1}\left(a_{1}, \ldots, a_{N} x_{a_{N}}\right)} K\left(a_{N}, b_{k}\right) \\
& \leq C_{0}^{5} C_{3} e^{\phi_{N-1}\left(a_{1}, \ldots, a_{N} x_{a_{N}}\right)} F\left(a_{N}, b_{k}\right) K\left(1_{\left[b_{k}\right]}, \omega\right) .
\end{aligned}
$$

So, for every $c \in S$ with $c \neq a_{i}$,

$$
\begin{aligned}
& K\left(1_{\left[a_{1}, \ldots, a_{N}\right]}, c\right) \geq C_{0}^{-1} e^{\phi_{N-1}\left(a_{1}, \ldots, a_{N} x_{a_{N}}\right)} K\left(a_{N}, c\right) \\
& (\because \text { Proposition A.2.7) } \\
& \geq C_{0}^{-3} e^{\phi_{N-1}\left(a_{1}, \ldots, a_{N} x_{a_{N}}\right)} \frac{F\left(a_{N}, c\right)}{F(o, c)} \\
& \text { ( } \because \text { Proposition A.2.1) } \\
& \geq C_{0}^{-4} e^{\phi_{N-1}\left(a_{1}, \ldots, a_{N} x_{a_{N}}\right)} \frac{F\left(a_{N}, b_{k}\right) F\left(b_{k}, c\right)}{F(o, c)} \\
& \text { ( } \because \text { Proposition A.2.2) } \\
& \geq C_{0}^{-6} e^{\phi_{N-1}\left(a_{1}, \ldots, a_{N} x_{a_{N}}\right)} F\left(a_{N}, b_{k}\right) K\left(b_{k}, c\right) \\
& (\because \text { Proposition A.2.1) } \\
& \geq C_{0}^{-11} C_{3}^{-1} K\left(\left[a_{1}, \ldots, a_{N}\right], b_{k}\right) \frac{K\left(b_{k}, c\right)}{K\left(1_{\left[b_{k}\right]}, \omega\right)} \quad(\because \text { equation (A.11)). }
\end{aligned}
$$


Let $\omega^{\prime} \in \mathcal{M}_{m}$ and let $c_{n} \in S$ with $\lim _{n \rightarrow \infty} K\left(f, c_{n}\right)=K\left(f, \omega^{\prime}\right)$ for every $f \in C_{c}(X)$. Such a sequence exists; see Proposition 2.3. Since equation (A.12) holds for all $n$ large enough,

$$
\mu_{\omega^{\prime}}\left(\left[a_{1}, \ldots, a_{N}\right]\right) \geq C_{0}^{-11} C_{3}^{-1} K\left(\left[a_{1}, \ldots, a_{N}\right], b_{k}\right) \frac{\mu_{\omega^{\prime}}\left(\left[b_{k}\right]\right)}{\mu_{\omega}\left(\left[b_{k}\right]\right)}
$$

Recall that for every $\mu \in \mathcal{C}$ there exists a finite measure $v$ on $\mathcal{M}_{m}$ such that $\mu=$ $\int K\left(\cdot, \omega^{\prime}\right) d \nu\left(\omega^{\prime}\right)$. Therefore, for every $\mu \in \mathcal{C}$ and every $f \in C_{c}^{+}(X)$ we have that

$$
\mu\left(\left[a_{1}, \ldots, a_{N}\right]\right) \geq C_{0}^{-11} C_{3}^{-1} K\left(\left[a_{1}, \ldots, a_{N}\right], b_{k}\right) \frac{\mu\left(\left[b_{k}\right]\right)}{\mu_{\omega}\left(\left[b_{k}\right]\right)} .
$$

Again, since the collection of cylinder sets linearly spans a dense subset of $C_{c}(X)$, the above inequality holds for all $f \in C_{c}^{+}(X)$. We take $k \rightarrow \infty$ and obtain that for every $\mu \in \mathcal{C}$ and every $f \in C_{c}^{+}(X)$,

$$
\mu(f) \geq C_{0}^{-11} C_{3}^{-1} \mu_{\omega}(f) \limsup _{k \rightarrow \infty} \frac{\mu\left(\left[b_{k}\right]\right)}{\mu_{\omega}\left(\left[b_{k}\right]\right)} .
$$

Let $\mu \in \mathcal{B}_{\omega}$ and let $\mu^{\prime}=\mu_{\omega}-\mu$. By definition of $\mathcal{B}_{\omega}, \mu^{\prime} \geq 0$ and so $\mu^{\prime} \in C$. Since $\inf _{f \in C_{c}^{+}(X)}\left(\mu^{\prime}(f) / \mu_{\omega}(f)\right)=0$, equation (A.13) with $\mu^{\prime}$ implies that $\lim _{k \rightarrow \infty}\left(\mu^{\prime}\left(\left[b_{k}\right]\right) /\right.$ $\left.\mu_{\omega}\left(\left[b_{k}\right]\right)\right)=0$. In particular, $\lim _{k \rightarrow \infty}\left(\mu\left(\left[b_{k}\right]\right) / \mu_{\omega}\left(\left[b_{k}\right]\right)\right)=1$. We use this fact and apply equation (A.13) with $\mu$ to obtain that $\mu \geq \epsilon_{2} \mu_{\omega}$ where $\epsilon_{2}=C_{0}^{-11} C_{3}^{-1} \in(0,1)$.

Set $c_{n}=\epsilon_{2}\left(1+\left(1-\epsilon_{2}\right)+\cdots+\left(1-\epsilon_{2}\right)^{n}\right)$. We show by induction that for every $\mu \in \mathcal{B}_{\omega}$ and every $n \geq 0, \mu \geq c_{n} \mu_{\omega}$. Since $c_{0}=\epsilon_{2}$, it is true for $n=0$. Let $n>0$ and suppose that $\mu \geq c_{k} \mu_{\omega}$ for every $\mu \in \mathcal{B}_{\omega}$ and every $k<n$. Then, for every $\mu \in \mathcal{B}_{\omega}$, $\left(\mu-c_{n-1} \mu_{\omega}\right) /\left(1-c_{n-1}\right) \in \mathcal{B}_{\omega}$ and so $\left(\mu-c_{n-1} \mu_{\omega}\right) /\left(1-c_{n-1}\right) \geq \epsilon_{2} \mu_{\omega}$. In particular, $\mu \geq\left(c_{n-1}+\epsilon_{2}\left(1-c_{n-1}\right)\right) \mu_{\omega}=c_{n} \mu_{\omega}$. Letting $n \rightarrow \infty$, we get that $\mu \geq \mu_{\omega}$. Therefore $\mu=\mu_{\omega}$ for every $\mu \in \mathcal{B}_{\omega}$, namely $\mathcal{B}_{\omega}=\left\{\mu_{\omega}\right\}$.

In what follows, let $\omega(\xi) \in \mathcal{M}_{m}$ be the unique limit point such that $K\left(\cdot, b_{n}\right) \rightarrow$ $K(\cdot, \omega(\xi))$, where $b_{n} \rightarrow \xi$ in the hyperbolic geometry. Since $\mathcal{A}_{\xi}$ contains a single point, $\omega(\xi)$ is well defined.

By Proposition 2.3 the mapping $\omega$ is onto. We show that for two boundary points $\xi, \eta \in$ $\partial S$ with $\eta \neq \xi$, we have that $K(\cdot, \omega(\xi)) \neq K(\cdot, \omega(\eta))$.

Lemma A.10. Let $(S, E)$ be a $\delta$-hyperbolic graph. Then, for two every boundary points $\xi, \eta \in \partial(S, E)$ there exists a two-sided infinite geodesic segment $\left(. \ldots, a_{-1}, a_{0}, a_{1}, \ldots\right)$ such that $a_{n} \underset{n \rightarrow \infty}{\longrightarrow} \xi$ and $a_{-n} \underset{n \rightarrow \infty}{\longrightarrow} \eta$.

Proof. See [54, Lemma 22.15].

By Proposition A.3.2 we have that for every $s \in S$,

$$
F(o, s)=C_{0}^{ \pm d_{E}\left(o, a_{0}\right)} F\left(a_{0}, s\right) .
$$


Hence, for every $n, k \geq 0$, with $C_{3}=C_{3}(0)$,

$$
\begin{aligned}
& \frac{K\left(a_{k}, a_{n}\right)}{K\left(a_{k}, a_{-n}\right)} \\
& \geq C_{0}^{-2} \frac{F\left(a_{k}, a_{n}\right) F\left(o, a_{-n}\right)}{F\left(o, a_{n}\right) F\left(a_{k}, a_{-n}\right)} \\
& \geq C_{0}^{-2-2 d_{E}\left(o, a_{0}\right)} \frac{F\left(a_{k}, a_{n}\right) F\left(a_{0}, a_{-n}\right)}{F\left(a_{0}, a_{n}\right) F\left(a_{k}, a_{-n}\right)} \\
& \geq C_{0}^{-2-2 d_{E}\left(o, a_{0}\right)} C_{3}^{-2} \frac{F\left(a_{k}, a_{n}\right) F\left(a_{0}, a_{-n}\right)}{F\left(a_{0}, a_{k}\right) F\left(a_{k}, a_{n}\right) F\left(a_{k}, a_{0}\right) F\left(a_{0}, a_{-n}\right)} \\
& =C_{0}^{-2-2 d_{E}\left(o, a_{0}\right)} C_{3}^{-2} \frac{1}{F\left(a_{0}, a_{k}\right) F\left(a_{k}, a_{0}\right)} \\
& \geq C_{0}^{-4-2 d_{E}\left(o, a_{0}\right)} C_{3}^{-2} \frac{G\left(a_{k}, a_{k}\right) G\left(a_{0}, a_{0}\right)}{G\left(a_{0}, a_{k}\right) G\left(a_{k}, a_{0}\right)} \\
& \geq C_{0}^{-4-2 d_{E}\left(o, a_{0}\right)} C_{3}^{-2} \frac{1}{G\left(a_{0}, a_{k}\right) G\left(a_{k}, a_{0}\right)} \\
& \left(\because G(a, a) \geq 1_{a}\left(a x_{a}\right)=1\right)
\end{aligned}
$$

Letting $n \rightarrow \infty$, we get that

$$
\frac{K\left(1_{\left[a_{k}\right]}, \omega(\xi)\right)}{K\left(1_{\left[a_{k}\right]}, \omega(\eta)\right)} \geq C_{0}^{-4-2 d_{E}\left(o, a_{o}\right)} C_{3}^{-2} \frac{1}{G\left(a_{0}, a_{k}\right) G\left(a_{k}, a_{0}\right)} .
$$

By Proposition A.2.6 and the assumption that $P_{G}(\phi)<0$, we have that

$$
\sum_{w \in S} G\left(a_{0}, w\right) G\left(w, a_{0}\right)<\infty .
$$

Therefore, $G\left(a_{0}, a_{k}\right) G\left(a_{k}, a_{0}\right) \rightarrow 0$ as $k \rightarrow \infty$. In particular, there exists $k$ such that $K\left(a_{k}, \omega(\xi)\right)>K\left(a_{k}, \omega(\eta)\right)$ and thus $\omega(\xi) \neq \omega(\eta)$.

\section{B. Appendix. Proof of Proposition 2.13}

Part (1) of the proposition was already proven in [21]. We prove part (2). Let $\mu$ be a Radon measure with $L_{\phi^{X, \delta}}^{*} \mu=\mu$ and assume by contradiction that $(\sigma, \gamma \Gamma) \in X$ is an atom. We first provide infinite sequences $\sigma^{n} \in \Sigma$ and $m_{n}, k_{n}>0$ such that

$$
T^{m_{n}}\left(\sigma^{n}, \gamma \Gamma\right)=T^{k_{n}}(\sigma, \gamma \Gamma) \text { for all } \gamma \in \Gamma_{0},
$$

and

$$
\exp \left(\phi_{m_{n}}^{\Sigma, \delta}\left(\sigma^{n}\right)-\phi_{k_{n}}^{\Sigma, \delta}(\sigma)\right)>C,
$$

where $C$ is a positive constant which does not depend on $n$.

For every $a, b \in S_{0}$ let $w_{a, b}$ be an admissible word that includes $b$ and such that $a w_{a, b} a$ is an admissible word. Since $(X, T)$ is topologically transitive, we can choose $w_{a, b}$ so that $e_{a w_{a, b}} \in \Gamma$. We also choose $w_{a, b}$ so that for every $a_{1}, a_{2} \in S_{0}$ with $a_{1} \neq a_{2}$, we have that $w_{a_{1}, b} \neq w_{a_{2}, b}$.

Let $\xi=\pi_{\Sigma}(\sigma)$ and let

$$
\sigma^{n, b}=\left(\sigma_{0}, \ldots, \sigma_{n-1}, \sigma_{n} w_{\sigma_{n}, b} \sigma_{n}, \sigma_{n+1}, \ldots\right) .
$$


For every $n$, let $b_{n} \in S_{0}$ such that $\pi_{\Sigma}\left(\sigma^{n, b_{n}}\right) \neq \xi$, let $\sigma^{n}=\sigma^{n, b_{n}}$ and let $\xi_{n}=\pi_{\Sigma}\left(\sigma^{n}\right)$. Such $b_{n}$ exists since $\left|S_{0}\right| \geq 4$ and $\pi_{\Sigma}$ is at most two-to-one (see [48]). Let $\alpha_{n} \in \Gamma_{0}$ such that $f_{\Gamma_{0}}^{n} \xi=\alpha_{n}^{-1} \xi$. By (Res), for all $n, f_{\Gamma_{0}}^{n} \xi_{n}=\alpha_{n}^{-1} \xi_{n}$. Let $l_{n}=\left|\sigma_{n} w_{\sigma_{n}, b_{n}}\right|$ and let $\beta_{n} \in \Gamma_{0}$ such that $f_{\Gamma_{0}}^{l_{n}}\left(f_{\Gamma_{0}}^{n} \xi_{n}\right)=\beta_{n}\left(f_{\Gamma_{0}}^{n} \xi_{n}\right)$. Since $f_{\Gamma_{0}}^{n} \xi=f_{\Gamma_{0}}^{n+l_{n}} \xi_{n}$, we have that

$$
\xi_{n}=\alpha_{n} \beta_{n}^{-1} \alpha_{n}^{-1} \xi
$$

Let $\gamma_{n}=\alpha_{n} \beta_{n}^{-1} \alpha_{n}^{-1}$. Observe that $\beta_{n}=e_{\sigma_{n} w_{\sigma_{n}, b_{n}}}^{-1} \in \Gamma$ and thus $\gamma_{n} \in \Gamma$. In particular, for all $\gamma \in \Gamma$,

$$
T^{n}(\sigma, \gamma \Gamma)=T^{n+l_{n}}\left(\sigma^{n}, \gamma \Gamma\right) .
$$

We deduce that

$$
\begin{aligned}
\left|\gamma_{n}^{\prime}(\xi)\right| & =\left|\alpha_{n}^{\prime}\left(\beta_{n}^{-1} \alpha_{n}^{-1} \xi\right)\right| \cdot\left|\left(\beta_{n}^{-1}\right)^{\prime}\left(\alpha_{n}^{-1} \xi\right)\right| \cdot\left|\left(\alpha_{n}^{-1}\right)^{\prime}(\xi)\right| \\
& =\left|\left(\beta_{n}^{-1}\right)^{\prime}\left(\alpha_{n}^{-1} \xi\right)\right| \cdot \frac{\left|\left(\alpha_{n}^{-1}\right)^{\prime}(\xi)\right|}{\left|\left(\alpha_{n}^{-1}\right)^{\prime}\left(\alpha_{n} \beta_{n}^{-1} \alpha_{n}^{-1} \xi\right)\right|} \\
& =\left|\left(\beta_{n}^{-1}\right)^{\prime}\left(f_{\Gamma_{0}}^{n} \xi\right)\right| \cdot \frac{\left|\left(f_{\Gamma_{0}}^{n}\right)^{\prime}(\xi)\right|}{\left|\left(f_{\Gamma_{0}}^{n}\right)^{\prime}\left(\xi_{n}\right)\right|} .
\end{aligned}
$$

By (Dist), there exists $B>1$ such that

$$
\frac{\left|\left(f_{\Gamma_{0}}^{n}\right)^{\prime}(\xi)\right|}{\left|\left(f_{\Gamma_{0}}^{n}\right)^{\prime}\left(\xi_{n}\right)\right|} \geq B^{-1} .
$$

Then, with

$$
D=\min _{a, b \in S_{0}} \min _{\xi^{\prime} \in[a]}\left|\left(e_{a w_{a, b}}^{-1}\right)^{\prime}\left(\xi^{\prime}\right)\right|>0,
$$

we have that

$$
\left|\gamma_{n}^{\prime}(\xi)\right| \geq \frac{D}{B}
$$

We show that $\left|\left\{\sigma^{n}\right\}\right|=\infty$. The mapping $\pi$ is continuous and thus $\xi_{n} \rightarrow \xi$. Since $\xi_{n} \neq \xi$ for all $n$, there exists a sub-sequence $\xi_{n_{k}}$ with $\left|\left\{\xi_{n_{k}}\right\}\right|=\infty$.

Finally, since

$$
\exp \left(\phi_{n}^{\Sigma, \delta}(\sigma)\right)=\left|\left(\alpha_{n}^{-1}\right)^{\prime}(\xi)\right|^{-\delta}
$$

and

$$
\exp \left(\phi_{n+l_{n}}^{\Sigma, \delta}\left(\sigma^{n}\right)\right)=\left|\left(\beta_{n} \alpha_{n}^{-1}\right)^{\prime}\left(\xi_{n}\right)\right|^{-\delta}=\left|\left(\alpha_{n} \beta_{n}^{-1}\right)^{\prime}\left(\alpha_{n}^{-1} \xi\right)\right|^{\delta},
$$

equation (A.1) follows with $k_{n}=n$ and $m_{n}=n+l_{n}$.

Next, observe that if $x, y \in X$ with $T^{n} x=T^{m} y$, then

$$
e^{-\phi_{n}^{X, \delta}(x)} \mu(\{x\})=e^{-\phi_{m}^{X, \delta}(y)} \mu(\{y\}) .
$$

Then, for every $\gamma \in \Gamma_{0}$,

$$
\mu\left(\left\{\left(\sigma^{n}, \gamma \Gamma\right)\right\}_{n \in \mathbb{N}}\right)=\sum_{n} \exp \left(\phi_{m_{n}}^{\Sigma, \delta}\left(\sigma^{n}\right)-\phi_{k_{n}}^{\Sigma, \delta}(\sigma)\right) \mu(\{x\})=\infty
$$

which contradicts the fact that $\mu$ is a Radon measure. 


\section{REFERENCES}

[1] J. Aaronson. An Introduction to Infinite Ergodic Theory (Mathematical Surveys and Monographs, 50). American Mathematical Society, Providence, RI, 1997.

[2] R. Adler and L. Flartto. Geodesic flows, interval maps, and symbolic dynamics. Bull. Amer. Math. Soc. (N.S.) 25(2) (1991), 229-334.

[3] A. Ancona. Negatively curved manifolds, elliptic operators, and the Martin boundary. Ann. Math. 125(3) (1987), 495-536.

[4] A. Ancona. Positive harmonic functions and hyperbolicity. Potential Theory Surveys and Problems. Springer, New York, NY, 1988, pp. 1-23

[5] M. Babillot. On the classification of invariant measures for horosphere foliations on nilpotent covers of negatively curved manifolds. Random Walks and Geometry. Walter de Gruyter, Berlin, 2004, pp. 319-335.

[6] T. Bedford, M. Keane and C. Series. Ergodic Theory, Symbolic Dynamics, and Hyperbolic Spaces. Oxford University Press, Oxford, UK, 1991.

[7] J. S. Birman and C. Series. Dehn's algorithm revisited, with applications to simple curves on surfaces. Combinatorial Group Theory and Topology (Utah, 1984). Ed. D. M. Gersten and J. R. Stallings. Princeton University Press, Princeton, NJ, 1987, pp. 451-478.

[8] S. R. P. Bispo and M. Stadlbauer. The Martin boundary of an extension by a hyperbolic group. Preprint, 2020, arXiv:2005.03723.

[9] S. Blachère, P. Haïssinsky and P. Mathieu. Harmonic measures versus quasiconformal measures for hyperbolic groups. Ann. Sci. Éc. Norm. Supér. (4) 44(4) (2011), 683-721.

[10] R. Bowen. Equilibrium States and the Ergodic Theory of Anosov Diffeomorphisms (Lecture Notes in Mathematics, 470). Springer, New York, NY, 1975.

[11] R. Bowen and C. Series. Markov maps associated with Fuchsian groups. Publ. Math. Inst. Hautes Études Sci. 50(1) (1979), 153-170.

[12] M. Burger. Horocycle flow on geometrically finite surfaces. Duke Math. J. 61(3) (1990), 779-803.

[13] D. Constantine, J. F. Lafont and D. J. Thompson. Strong symbolic dynamics for geodesic flow on CAT $(-1)$ spaces and other metric Anosov flows. J. Éc. Polytech. Math. 7 (2020), 201-231.

[14] M. Coornaert. Mesures de Patterson-Sullivan sur le bord d'un espace hyperbolique au sens de Gromov. Pacific J. Math. 159(2) (1993), 241-270.

[15] R. Coulon, F. Dal'Bo, and A. Sambusetti. Growth gap in hyperbolic groups and amenability. Geom. Funct. Anal. 28(5) (2018), 1260-1320.

[16] S. G. Dani. Invariant measures of horospherical flows on noncompact homogeneous spaces. Invent. Math. 47(2) (1978), 101-138.

[17] P. de La Harpe. Topics in Geometric Group Theory. University of Chicago Press, Chicago, IL, 2000.

[18] M. Denker and M. Urbański. On the existence of conformal measures. Trans. Amer. Math. Soc. 328(2) (1991), 563-587.

[19] R. Dougall and R. Sharp. Amenability, critical exponents of subgroups and growth of closed geodesics. Math. Ann. 365(3-4) (2016), 1359-1377.

[20] P. Eberlein. Geodesic flow in certain manifolds without conjugate points. Trans. Amer. Math. Soc. 167 (1972), 151-170.

[21] K. Falk, K. Matsuzaki and B. Stratmann. Checking atomicity of conformal ending measures for Kleinian groups. Conform. Geom. Dyn. 14(8) (2010), 167-183.

[22] H. Furstenberg. The unique ergodigity of the horocycle flow. Recent Advances in Topological Dynamics. Springer, New York, NY, 1973, pp. 95-115.

[23] É. Ghys and P. de La Harpe. Sur les groupes hyperboliques d'apres Mikhael Gromov (Progress in Mathematics, 83). Birkhäuser Boston, Inc., Boston, 1990.

[24] S. Gouëzel. Local limit theorem for symmetric random walks in Gromov-hyperbolic groups. J. Amer. Math. Soc. 27(3) (2014), 893-928.

[25] S. Gouëzel and S. P. Lalley. Random walks on co-compact Fuchsian groups. Ann. Sci. Éc. Norm. Supér. (4) 46 (2013), 131-175.

[26] J. Jaerisch. Group-extended Markov systems, amenability, and the Perron-Frobenius operator. Proc. Amer. Math. Soc. 143(1) (2015), 289-300.

[27] V. A. Kaimanovich. Ergodicity of harmonic invariant measures for the geodesic flow on hyperbolic spaces. J. Reine Angew. Math. 455 (1994), 57-104.

[28] V. A. Kaimanovich. Ergodic properties of the horocycle flow and classification of Fuchsian groups. J. Dyn. Control Syst. 6(1) (2000), 21-56.

[29] A. Karlsson and F. Ledrappier. On laws of large numbers for random walks. Ann. Probab. 34(5) (2006), 1693-1706. 
[30] A. Karlsson and G. A. Margulis. A multiplicative ergodic theorem and nonpositively curved spaces. Commun. Math. Phys. 208(1) (1999), 107-123.

[31] F. I. Karpelevič. The geometry of geodesics and the eigenfunctions of the Beltrami-Laplace operator on symmetric space. Trans. Moscow Math. Soc. (1965), 51-199.

[32] O. Landesberg and E. Lindenstrauss. On Radon measures invariant under horospherical flows on geometrically infinite quotients. Preprint, 2019, arXiv:1910.08956.

[33] F. Ledrappier and O. Sarig. Invariant measures for the horocycle flow on periodic hyperbolic surfaces. Israel J. Math. 160(1) (2007), 281-315.

[34] T. Lyons and D. Sullivan. Function theory, random paths and covering spaces. J. Differential Geom. 19(2) (1984), 299-323.

[35] D. Mauldin and M. Urbański. Gibbs states on the symbolic space over an infinite alphabet. Israel J. Math. 125(1) (2001), 93-130.

[36] P. J. Nicholls. The Ergodic Theory of Discrete Groups (London Mathematical Society Lecture Note Series, 143). Cambridge University Press, Cambridge, MA, 1989.

[37] S. J. Patterson. The limit set of a Fuchsian group. Acta Math. 136(1) (1976), 241-273.

[38] M. Rees. Checking ergodicity of some geodesic flows with infinite Gibbs measure. Ergod. Th. \& Dynam. Sys. 1(1) (1981), 107-133.

[39] T. Roblin. Ergodicité et équidistribution en courbure négative. Société Mathématique de France, Paris. 2003.

[40] T. Roblin. Comportement harmonique des densités conformes et frontière de Martin. Bull. Soc. Math. France 139(1) (2011), 97-127.

[41] O. Sarig. Thermodynamic formalism for countable Markov shifts. Ergod. Th. \& Dynam. Sys. 19(06) (1999), 1565-1593.

[42] O. Sarig. Thermodynamic formalism for null recurrent potentials. Israel J. Math. 121(1) (2001), $285-311$.

[43] O. Sarig. The horocycle flow and the Laplacian on hyperbolic surfaces of infinite genus. Geom. Funct. Anal. 19(6) (2010), 1757-1812.

[44] O. Sarig. Thermodynamic formalism for countable Markov shifts. Proc. Sympos. Pure Math. 89 (2015), 81-117.

[45] O. Sarig and B. Schapira. The generic points for the horocycle flow on a class of hyperbolic surfaces with infinite genus. Int. Math. Res. Not. IMRN 2008 (2008), rnn086.

[46] C. Series. The infinite word problem and limit sets in Fuchsian groups. Ergod. Th. \& Dynam. Sys. 1(3) (1981), 337-360.

[47] C. Series. Martin boundaries of random walks on Fuchsian groups. Israel J. Math. 44(3) (1983), $221-242$.

[48] C. Series. Geometrical Markov coding of geodesics on surfaces of constant negative curvature. Ergod. Th. \& Dynam. Sys. 6(4) (1986), 601-625.

[49] O. Shwartz. Thermodynamic formalism for transient potential functions. Commun. Math. Phys. 366(2) (2019), 737-779.

[50] M. Stadlbauer. An extension of Kesten's criterion for amenability to topological Markov chains. Adv. Math. 235 (2013), 450-468.

[51] M. Stadlbauer. On conformal measures and harmonic functions for group extensions. New Trends in One-dimensional Dynamics. Springer, New York, NY, 2016.

[52] D. Sullivan. The density at infinity of a discrete group of hyperbolic motions. Publ. Math. Inst. Hautes Études Sci. 50(1) (1979), 171-202.

[53] D. Sullivan. Related aspects of positivity in Riemannian geometry. J. Differential Geom. 25(3) (1987), 327-351.

[54] W. Woess. Random Walks on Infinite Graphs and Groups (Cambridge Tracts in Mathematics, 138). Cambridge University Press, Cambridge, MA, 2000. 